\title{
Moment-Based Analysis of Reg. Triangular QAM in Turbulent Atmospheric Channels with Pointing Errors incorporates Zero and Non-zero Boresight
}

\section{Muhammad Nabeel Shahid}

National University of Sciences and Technology

Furqan Haider Qureshi ( $\nabla$ furqan71@ee.ceme.edu.pk)

National University of Sciences and Technology https://orcid.org/0000-0003-0481-7459

Shahzad Amin Sheikh

National University of Sciences and Technology

Qasim Umar Khan

National University of Sciences and Technology

Muhammad Zeeshan

National University of Sciences and Technology

\section{Research}

Keywords: Quadrature amplitude modulation, optical communication, Rayleigh Channels, Moment methods, Log normal distribution, Rician channels, Error analysis.

Posted Date: October 5th, 2020

DOl: https://doi.org/10.21203/rs.3.rs-72284/v1

License: (c) (1) This work is licensed under a Creative Commons Attribution 4.0 International License. Read Full License 


\title{
Moment-Based Analysis of Reg. Triangular QAM in Turbulent Atmospheric Channels with Pointing Errors incorporates Zero and Non-zero Boresight
}

\author{
Muhammad Nabeel Shahid ${ }^{1}$ \\ Email: nabeel.shahid16@ee.ceme.edu.pk
}

Furqan Haider Qureshi ${ }^{1, *}$

Email: furqan71@ee.ceme.edu.pk

Shahzad Amin Sheikh ${ }^{1}$

Email: sheikh.shahzadamin@gmail.com

Qasim Umar Khan ${ }^{1}$

Email: qasimumarkhan@yahoo.com

Muhammad Zeeshan ${ }^{1}$

Email: ranazeeshan@ceme.nust.edu.pk

${ }^{1}$ (Department of Electrical Engineering, College of Electrical and Mechanical Engineering, National University of Sciences and Technology, Islamabad, Pakistan.)

*Corresponding author 


\section{Abstract}

In recent years, free-space optical (FSO) technology has gained fame in communication systems due to its high data rates and license-free feature. Triangular quadrature amplitude modulation (TQAM) is an efficient modulation scheme that uses even bits per symbol, and it has a low average symbol error rate (ASER) than square quadrature amplitude modulation (SQAM). In this paper, we theoretically investigate the performance of subcarrier triangular quadrature amplitude modulation (SC-TQAM) corrupted by atmospheric turbulence in the presence of pointing errors (P.E) in FSO communications. We have considered boresight displacement, P.E effect, and atmospheric turbulence. To represent the atmospheric turbulence we consider Log-normal, Rayleigh and Rician distributions which exhibit weak, moderate and strong weather effects. The P.E is employed using Rayleigh and Rician distribution that incorporates zero and non-zero boresight displacement respectively. The moment generating functions of these models have been derived. P.E is modeled using an optical beam radius and receiver aperture radius. The combination of these parameters will help enhance the average symbol error rate (ASER). Furthermore, we have derived an analytical expression that is used to develop numerical results. The ASER performance is observed against average received irradiance, optical beam radius, P.E standard deviation and receiver aperture radius. In the end, the ASER performance is evaluated against SNR and Monte Carlo simulations are performed which validates the theoretical results.

Keywords: Quadrature amplitude modulation, optical communication, Rayleigh Channels, Moment methods, Log normal distribution, Rician channels, Error analysis. 


\section{Introduction}

Over the last two decades, FSO technology is readily earning fame as an efficient channel of transmitting data over short distances at high speed. FSO systems are presently developing as a replacement of fiber optics to overcome the huge bandwidth requirements. This is above all owing to exceptional attributes of the FSO technology, which comprise: almost endless bandwidth for acquiring very high accumulated capacity, cost-efficient, reduced intrusion, no licensing requirements, and highly secure link that is simple to design and implement $[1,2]$. FSO has already shown the superiority of high bandwidth utilization and zero electromagnetic Interference (EMI) [3].

The license-free feature of FSO systems has significant importance as the Radiofrequency spectrum is getting congested due to an increase in the number of users every day. Although FSO has considerable potential to fix the last mile problems, FSO communications is the least deployed technology among the others due to many problems. These problems are primarily induced due to weather conditions viz. snow, fog, and rain. Atmospheric turbulence effect on a clear day has a considerable effect on the performance of FSO systems. It is a line of sight (LOS) communication utilizing wavelengths of different frequencies viz. 850nm, 1300nm and 1550nm [4].

The phenomenon of atmospheric turbulence occurs when the refractive index of channel i.e. air

or free space is altered due to change in pressure and temperature. Therefore, the turbulent channel which is extremely fickle, random and exposed to weather conditions causes scattering and absorption which leads to severe errors in received signal [5]. 
Besides atmospheric turbulence, P.E also affects optical links performance that adds more fading at the receiver's end. Pointing error is a phenomenon that is observed at the receiver due to building sway and transceiver misalignment, which causes loss in terms of power and induce fading in the received signal. As a result, transceiver misalignment causes a random shift in the LASER beam footprint along the vertical and horizontal axis. Bore-sight and jitters are two main components of pointing errors. The offset induced in the beam center at the receiver's aperture is known as jitter and displacement of beam footprint in $\mathrm{X}-\mathrm{Y}$ plane on receiver's aperture is known as bore-sight [6].

Many probabilistic models have been suggested to incorporate the effect of atmospheric turbulence as weak, moderate and strong intensity fluctuations. The log-normal statistical model is used to describe weak-to-moderate, while gamma-gamma represents strong fading regime [7]. Over the past few years, a number. of generalized pointing error, models have been presented. In [8], a generalized P.E model is proposed that incorporates zero bore-sight and similar jitter in elevation and azimuth directions. In [9], a more realistic approach is considered that incorporates the effect of wind and thermal expansion as jitter variances in terrestrial FSO Links.

In FSO, the most used modulation scheme is on-off keying (OOK), due to its simplicity and less cost demanding features. However, opting for the right value of the adaptive threshold in OOK modulation is hard to achieve, due to fluctuation intensity in the signal [10]. As compare to OOK, pulse position modulation (PPM) has exceptional power and poor spectral efficiency [11]. Multiple phase-shift keying (MPSK) and multiple pulse amplitude modulation (MPAM) gives better performance then OOK $[12,13]$. 
Other than PSK, intensity modulation (IM) with quadrature amplitude modulation (QAM) has gained popularity in recent years. A $2^{N}$ QAM transmits $N$ times more bits than binary phase keying (BPSK), where $N=\log _{2}(M)$. The S-QAM modulation scheme that uses even bits per symbol $M=2^{2}, 2^{4}, 2^{6}, \ldots$ and X-QAM is optimum modulation scheme that uses odd bits per symbol $M=2^{3}, 2^{5}, 2^{7}, \ldots$ since XQAM has low symbol error probability than R-QAM [14, 15].

A hexagonal constellation was presented that has a structure similar to honeycomb (HCSS) was presented, another constellation named as triangular shape signal set (TSSS) was proposed. HCSS and TSSS both performs better than SQAM, but they have high receiver detection complexity $[16,17]$. In [18], triangular quadrature constellation was proposed, the constellation points were taken on the vertices of the regular polygon. TQAM, as compare to SQAM, is efficient in terms of performance and receiver detection complexity $[18,19]$. TQAM transmits even bits encoded data and due to its compact geometry, it performs better than SQAM [20]. In [21], an approximate SEP expression for TQAM was derived and also, In [22, 23], an exact SEP expression was proposed and its performance evaluated over various fading channels in RF communication. It is noticed from the afore discussed literature, that to the best of authors' knowledge no research has been conducted on performance evaluation of TQAM over Freespace communication.

Hence, our motivation is to derive statistical models, probability density function (PDF), for TQAM which models the fading effect of the turbulent channel and pointing errors considering transmitter beam radius, jitter standard deviation, and photodetector aperture. Analytical expression for general order TQAM has been utilized, the effect of transmitter beam-width, jitter's standard deviation and photodetector aperture on ASER performance is observed. The mathematical ASER performance is evaluated and validated via Monte Carlo simulation. 
Moreover, the impact of previously discussed factors e.g. jitter's standard deviation $\sigma_{s}$ and transmitter beam radius $\omega_{0}$ is used to optimize ASER performance.

This paper comprises of the following sections: In section 2 we will discuss system model, in section 3 fading channels are discussed, in section 4 statistical models are derived along with their moment generating functions, in section 5 expression for ASER is presented, in section 6 numerical results are discussed, and conclusion is given along with future recommendations in section 7.

\section{SYSTEM MODEL AND MODULATION}

A system using general order TQAM is considered and, the source generates the data which is pre-modulated to radio RF can be written as [24].

$$
k_{n}(t)=\mathrm{J}_{\mathrm{I}, \mathrm{n}} \cos \left(2 \pi f_{c} t\right)+\mathrm{J}_{\mathrm{Q}, \mathrm{n}} \sin \left(2 \pi f_{c} t\right), \quad 0 \leq t \leq T_{s}
$$

Where $\mathrm{J}_{\mathrm{I}, \mathrm{n}}$ and $\mathrm{J}_{\mathrm{Q}, \mathrm{n}}$ are the amplitudes of in-phase and quadrature components respectively, $f_{c}$ is the carrier frequency and $T_{s}$ is the symbol period. The SC-TQAM is used to derive the LASER diode circuit as the SC-TQAM varies and generates a modulated signal in terms of irradiance. The generalized transmitted signal is written as

$$
s(t)=I_{0}\left[1+\varsigma\left[J_{\mathrm{I}, \mathrm{n}} \cos \left(2 \pi f_{c} t\right)+\mathrm{J}_{\mathrm{Q}, \mathrm{n}} \sin \left(2 \pi f_{c} t\right)\right]\right]
$$

where $I_{0}$ is the average irradiance power, $\varsigma$ is the modulation index and $s(t)$ is the transmitted signal. The SC-TQAM signal is used to modulate the intensity of LASER, with proper biasing to avoid any chopping, the LASER beam is aligned with the receiver using a collimator (telescope) and transmitted over the channel. The received signal is expressed as 


$$
y(t)=I_{0} H+I_{0} H_{\varsigma}\left[J_{\mathrm{I}, \mathrm{n}} \cos \left(2 \pi f_{c} t\right)+\mathrm{J}_{\mathrm{Q}, \mathrm{n}} \sin \left(2 \pi f_{c} t\right)\right]
$$

where $H$ is the scaling factor of channel and it temper's the signal due to the presence of atmospheric turbulence and pointing errors. The $I_{0} H$ is DC noise and it can be filtered by using a band-pass filter. The LASER beam distorted by atmospheric turbulence

Figure 1 Basic block diagram of FSO communication system

is collected at the receiver's aperture, focused on the photo-detector (PD) generates an electrical signal. The signal recovered at photo-detector can be written as

$$
y_{k}(t)=H \varsigma I_{0} R k_{n}(t)+v(t)
$$

where $R$ is the responsitivity of photo-detector and $v(t)$ can be modeled as white Gaussian noise (AWGN). The generalized expression for equation (4) can be written as

$$
y=H x+v
$$

where $H$ is the received signal, $x$ is the transmitted symbol, $H$ is the scaling factor of channel used and $v$ is receiver noise which is modeled as white Gaussian noise (AWGN) having a spectral density $N_{0}$. Assuming the scaling factor of fading channel $H$ is accurately known to receiver, the electrical signal is TQAM demodulated and implementing equalization can be written as

$$
Y=\frac{y}{H}=\frac{(H \times x+v)}{H}
$$

where $Y$ is the recovered signal, which is compared with transmitted signal $x$, symbol error rate (SER) can be computed as 


$$
P_{e}=\frac{N_{e}}{N}
$$

where $P_{e}$ is the probability of symbol error, $N_{e}$ is total no. of symbols received in error and $N$ represents the total no. of symbols transmitted.

\section{Fading Channels}

Free-space optical (FSO) system is a technology that combines two different types of communication i.e. optical fiber and wireless communication. The optical beam is transmitted in the atmosphere (Channel), to recover transmitted signal at the receiver, a mathematical model is required to be formulated. This light signal experience attenuation due to the turbulent nature of the channel. The particles present in the atmosphere act as a barrier; reflect, absorb, refract, attenuate, disperse and diffract the optical beam [24]. The phenomena of dispersion happen when a signal is transmitted, it reaches the receiver at different time intervals through multiple paths. If the terminals of transceiver are relatively in motion it is dispersion in frequency. Otherwise, if transmitter and receiver are stationary it is dispersion in time [25]. The phenomena of fading can be classified as follows: Shadowing and multi-path fading. Shadowing referred to fading induced due to reflection, diffraction and absorption from small stationary and non-stationary obstacles e.g. buildings, vehicles, and trees. These obstacles cause small variations in mean intensity of signal amplitude. In shadowing, the coherence time is larger than symbol time period $T_{c}>T_{s}$. The effect of shadowing can be completely removed by employing specialized systems. In contrary to that, the multipath fading or small-scale fading is occurred when signal reaches at receiver at different times reflecting through obstacles or reaches directly from the transmitter. These delayed multipath adds up at receiver and behaves constructively or destructively. Moreover, in this scenario the coherence time is smaller than symbol time period $T_{c}<T_{S}$ [25]. In 
the subsequent section, to study the effect of different fading conditions; Log-Normal as shadowing; Rayleigh and Rician as multipath fading has been employed.

\section{A. Log-Normal Shadowing}

Log-Normal Shadowing is utilized when the particles present in the air are almost frozen. It is a normal practice to model path loss using Log-Normal distribution in multi path fading. When a signal propagates through channel, it gets corrupted due to reflection, refraction and diffraction. When all possible losses are added to together, according to central limit theorem it tends to follow Log-Normal distribution [26]. The random variable I can be modeled as Log-Normal distribution. In FSO communication, Log-Normal is used to illustrate the weak atmospheric fading effect. The PDF of Log-Normal distribution is expressed as

$$
f_{I}(I)=\frac{1}{I \sqrt{2 \pi \sigma_{I}^{2}}} \exp \left(-\frac{\left(\ln \left(\frac{I}{I_{o}}\right)+\frac{1}{2 \sigma_{I}^{2}}\right)^{2}}{2 \sigma_{I}^{2}}\right)
$$

where $I$ is the signal intensity level, $\sigma_{\mathrm{I}}^{2}=0.31 k^{\frac{7}{6}} C_{n}^{2} z^{\frac{11}{6}}$ is $\log$ amplitude variance, $I_{o}$ is mean received irradiance, the relation of log-amplitude variance with Raytov variance is $\sigma_{I}^{2} \approx \sigma_{R}^{2}, \sigma_{R}^{2}$ is Raytov variance. $k=2 \pi \lambda$ is the optical wave no. and $\lambda$ is the wavelength.

In a communication system, the symbol error rate is a key parameter to analyze the performance of the modulation scheme in any channel. Now, we will derive a moment generating function (MGF) that will be utilized to investigate symbol error performance. The generalized mathematical expression of (MGF) is given as [25] 


$$
I(\beta, \varphi)=2 \int_{0}^{\infty} Q(\beta, \varphi) f(I) d I
$$

where $\beta=\sqrt{2 D \gamma(I)}, \gamma(I)$ is electrical (SNR) per symbol and $f(I)$ is statistical model of channel. The (SNR) per symbol is expressed as $\gamma(I)=\frac{2 R^{2} I^{2} \zeta^{2}}{4 \sigma_{I}^{2}}$, where $R$ is responsivity of receiver and \$zeta is modulation index. By substituting $R=1$ and $\zeta=1$ the expression of electrical (SNR) per symbol reduces to $\gamma(I)=\frac{I^{2}}{\sigma_{I}^{2}}$. Now, in order to derive (MGF) for M-TQAM substituting the equation (8) in equation (9), we get

$$
I(\beta, \varphi)=2 \int_{0}^{\infty} Q(\beta, \varphi) \frac{1}{I \sqrt{2 \pi \sigma_{I}^{2}}} \exp \left(-\frac{\left(\ln \left(\frac{I}{I_{o}}\right)+\frac{1}{2 \sigma_{I}^{2}}\right)^{2}}{2 \sigma_{I}^{2}}\right) d I
$$

Now, supposing $x^{2}=-\frac{\left(\ln \left(\frac{I}{I_{0}}\right)+\frac{1}{2 \sigma_{I}^{2}}\right)^{2}}{2 \sigma_{I}^{2}}$ and rearranging expression of $x^{2}$ in terms of $I$ the equation becomes

$$
I=I o \exp \left(\sqrt{2 \sigma_{I}^{2}} x-\frac{\sigma_{I}^{2}}{2}\right)
$$

substituting the $x^{2}$ in equation (10), we get

$$
I(\beta, \varphi)=2 \int_{0}^{\infty} Q(\beta, \varphi) \frac{1}{I \sqrt{2 \pi \sigma_{I}^{2}}} \exp \left(-x^{2}\right) d x
$$


to reduces equation (12) to close form expression, we will use Gauss Hermite Quadrature approximation stated in [24], which is stated as

$$
\int_{0}^{\infty} f(x) e^{-x^{2}}=\sum_{i=0}^{\infty} \omega_{i} f\left(x_{i}\right)
$$

where $x_{i}$ is zeros and $\omega_{i}$ is the weight factors. considering $Q(\beta, \varphi)=f\left(x_{i}\right)$ and replacing $e^{-x^{2}}=\omega_{i}$ in equation (13). Furthermore, alternate form of Q-function is utilized which is also known as Craig's form $\left(\phi=\frac{\pi}{2}\right)$ expressed as [27, 28]

$$
Q(x, \varphi)=\frac{1}{\pi} \int_{0}^{\varphi} \exp \left(-\frac{x^{2}}{2 \sin ^{2} \theta}\right) d \theta, x \geq 0
$$

Now substituting Craig's form in equation (12), new expression becomes

$$
I(\beta, \varphi)=\frac{1}{\sqrt{\pi}} \sum_{i=0}^{\infty} \frac{1}{\pi} \omega_{i} \int_{0}^{\varphi} \exp \left(-\frac{2 D \gamma}{2 \sin ^{2} \theta}\right) d \theta
$$

now substituting $\gamma=\frac{I^{2}}{2 \sigma_{I}^{2}}$ in equation (15), we get

$$
I(\beta, \varphi)=\frac{1}{\sqrt{\pi}} \sum_{i=0}^{\infty} \frac{1}{\pi} \omega_{i} \int_{0}^{\varphi} \exp \left(-\frac{2 D \frac{I^{2}}{2 \sigma_{I}^{2}}}{2 \sin ^{2} \theta}\right) d \theta
$$

now substituting in equation (11) in equation (16) 


$$
I(\beta, \varphi)=\frac{2}{\sqrt{\pi}} \sum_{i=0}^{\infty} \frac{1}{\pi} \omega_{i} \int_{0}^{\varphi} \exp \left(-\frac{2 D\left(\frac{I_{o}{ }^{2}}{2 \sigma_{I}^{2}}\right)\left(\exp \left(\sqrt{2 \sigma_{I}^{2}} x-\frac{\sigma_{I}^{2}}{2}\right)\right)^{2}}{2 \sin ^{2} \theta}\right) d \theta
$$

Now, substituting $k_{0}=\frac{I_{0}^{2}}{2 \sigma_{I}^{2}}$ as different types of noise limiting conditions, now reducing equation (17) using Craig's form, we get the final expression as

$$
I(\beta, \varphi)=\frac{2}{\sqrt{\pi}} \sum_{i=0}^{\infty} \frac{1}{\pi} \omega_{i} Q\left(\sqrt{2 D k_{o}}\left(\exp \left(\sqrt{2 \sigma_{I}^{2}} x-\frac{\sigma_{I}^{2}}{2}\right)\right)\right)
$$

The mathematical expression shown above will be used in subsequent section. It will be utilized to evaluate the symbol error rate for M-TQAM in Log-Normal Channel.

\section{B. Rayleigh Fading Channel}

Rayleigh fading channel in strong turbulence regime incorporates stronger fluctuations in the amplitude of received signal irradiance. Its application is considered when a number of scatterers are present in the atmosphere, which scatters the transmitted signal. It involves no direct line of sight communication between transmitter and receiver. Normally, the Rayleigh fading channel is utilized when the scatterings are independent, severe in effect and the optical link spans a few kilometers. These independent scatterings are modeled as Gaussian. Rayleigh Fading is observed when the radius $(r)$ of particles present in the channel is smaller in size then wavelength $(\lambda)$ of LASER beam $(r<\lambda)$. The Rayleigh distribution is modeled using negative exponential distribution and its probability density function can be written as [29]. 


$$
f(I)=\frac{1}{I_{o}} \exp \left(-\frac{I}{I_{o}}\right)
$$

where $I$ is the signal intensity level and $I_{o}$ is mean received irradiance. Moreover, in saturation regime the scintillation index of strong turbulence is almost unity and it is also known as fully developed speckle regime. The Rayleigh channel is modeled using Gaussian random with zero mean. Thus, SNR per symbol is central chi-square distributed [25].

In order to derive the close form expression for $M G F$ to evaluate the symbol error rate performance of M-TQAM in Rayleigh channel. Now, substituting equation (19) and equation (15) in equation (9), we get

$$
I(D, \varphi)=\frac{2}{\pi I_{o}} \int_{0}^{\varphi} \int_{0}^{\infty} \exp \left(-\frac{2 D \gamma(I)}{2 \sin ^{2} \theta}\right) \exp \left(-\frac{I}{I_{o}}\right) d I d \theta
$$

substituting $\gamma(I)=\frac{I^{2}}{2 \sigma^{2}}$ and by approximating $I^{2}$ to $I$ in equation (20) can be written as

$$
I(D, \varphi)=\frac{2}{\pi I_{o}} \int_{0}^{\varphi} \int_{0}^{\infty} \exp \left(-\frac{2 D \frac{I^{2}}{2 \sigma^{2}}}{2 \sin ^{2} \theta}\right) \exp \left(-\frac{I}{I_{o}}\right) d I d \theta
$$

Now taking $-I$ common from the exponential term we get

$$
I(D, \varphi)=\frac{2}{\pi I_{o}} \int_{0}^{\varphi} \int_{0}^{\infty} \exp \left(-I\left(\frac{2 D \frac{1}{2 \sigma^{2}}}{2 \sin ^{2} \theta}+\frac{1}{I_{o}}\right)\right) d I d \theta
$$

By solving integral with variable $I$ we get 


$$
I(D, \varphi)=\frac{2}{\pi I_{o}} \int_{0}^{\varphi}\left|\frac{-e^{-I}}{\frac{2 D I_{o}}{2 \sigma^{2}}+2 \sin ^{2} \theta}\right|_{0}^{\infty} d \theta
$$

After applying the limits and simplifying the above expression we get

$$
I(D, \varphi)=\frac{2}{\pi I_{o}} \int_{0}^{\varphi} \frac{2 \sin ^{2} \theta}{\frac{2 D I_{o}}{2 \sigma^{2}}+2 \sin ^{2} \theta} d \theta
$$

where the $M G F$ of Rayleigh Fading channel is written as

$$
M_{I}\left(D, \frac{I_{o}}{2 \sigma^{2}}\right)=\frac{\sin ^{2} \theta}{\frac{D I_{o}}{2 \sigma^{2}}+\sin ^{2} \theta}
$$

After solving the integral in equation (24) and applying limit we get

$$
\begin{aligned}
I\left(D, \frac{I_{o}}{2 \sigma^{2}}\right) & =\frac{2}{\pi}\left[\frac{\pi}{2}-\frac{\pi}{2}\left[\sqrt{\frac{\frac{2 D I_{o}}{4 \sigma^{2}}}{\frac{2 D I_{o}}{4 \sigma^{2}}+1}}\right]\right] \\
& =1-\sqrt{\frac{\frac{D I_{o}}{2 \sigma^{2}}}{\frac{D I_{o}}{2 \sigma^{2}}+1}}
\end{aligned}
$$

The expression written above will be utilized in subsequent section for evaluating symbol error rate of M-TQAM in Rayleigh fading channel. 


\section{Rician Fading Channel}

In this channel signal propagates through multipaths and reaches the receiver at different time intervals. There is one direct line of sight component and the rest of components are delayed random reflections of the transmitted signal. The channel with specular and non-specular components can be mathematically formulated by Rician distribution function which is as follows $[24,25]$

$$
f_{r}(r)=\frac{e^{-n^{2}}\left(1+n^{2}\right)}{I_{o}} \exp \left(-\frac{I\left(1+n^{2}\right)}{I_{o}}\right) B_{o}\left(\sqrt{\frac{4 n^{2}\left(1+n^{2}\right) I}{I_{o}}}\right)
$$

where $I$ is the signal intensity, $I_{0}$ is mean received irradiance, $B_{0}$ refers to zeroth order modified Bessel function of first kind and $n^{2}$ is K-factor of Rician distribution.It is noteworthy that Rician distribution is modeled using Gaussian random variables having non zero mean. Therefore, the SNR per symbol is non-central chi-square distributed.

Now, we will develop a close form mathematical expression to evaluate the symbol error rate in Rician Fading channel for M-TQAM. To develop the expression, we will substitute equation (14) in equation (9) we get

$$
\begin{gathered}
I(D, \varphi)=\frac{2}{\pi} \int_{0}^{\varphi} \int_{0}^{\infty} \exp \left(-\frac{2 D \gamma(I)}{2 \sin ^{2} \theta}\right) f(I) d I d \theta \\
=\frac{2}{\pi} \int_{0}^{\varphi}\left[\int_{0}^{\infty} \exp \left(-\frac{2 D \gamma(I)}{2 \sin ^{2} \theta}\right) f(I) d I\right] d \theta
\end{gathered}
$$


In equation (28), the term written in square brackets can be solved using Laplace transform. The generalized expression for Laplace transform is written as

$$
M_{I}(\beta)=\int_{0}^{\infty} e^{\beta I} f(I) d I
$$

Now, rewriting the equation (28) in terms of Laplace transform we get

$$
I(D, \varphi)=\frac{2}{\pi} \int_{0}^{\varphi} M_{I}\left(\frac{-2 D}{2 \sin ^{2} \theta}\right) d \theta
$$

The mathematical expression written within square brackets in equation (28) is directly taken from [25] and written as

$$
M_{I}\left(\frac{2 D}{2 \sin ^{2} \theta}\right)=\frac{(1+K) 2 \sin ^{2} \theta}{(1+K) 2 \sin ^{2} \theta+D \frac{I_{0}^{2}}{\sigma^{2}}} \exp \left(-\frac{K D \frac{I_{0}^{2}}{\sigma^{2}}}{(1+K) 2 \sin ^{2} \theta+D \frac{I_{0}^{2}}{\sigma^{2}}}\right)
$$

where $K=n^{2}$ that is Rician K-factor, by substituting the above expression in equation (30) we get the final expression

$$
I(D, \varphi)=\frac{2}{\pi} \int_{0}^{\varphi} \frac{(1+K) 2 \sin ^{2} \theta}{(1+K) 2 \sin ^{2} \theta+D \frac{I_{0}^{2}}{\sigma^{2}}} \exp \left(-\frac{K D \frac{I_{0}^{2}}{\sigma^{2}}}{(1+K) 2 \sin ^{2} \theta+D \frac{I_{0}^{2}}{\sigma^{2}}}\right) d \theta
$$

\section{Atmospheric turbulence model with pointing errors}

In this section we will derive the composite channel model recalling the method stated in [4]. The channel gain $\$ \mathrm{H} \$$ is characterized by three factors viz. atmospheric turbulence $H_{a}$, 
atmospheric attenuation $H_{l}$ and pointing errors $H_{p}$. When optical signal passes through turbulent channel it gets distorted due to rain, fog and dust. The channel gain $H$ can be expressed as [8]

$$
H=H_{p} \times H_{a} \times H_{l}
$$

where, $H_{l}$ is a deteministic quantity exhibits no randomness, it is expressed as

$$
H_{l}=\exp (-\sigma z)
$$

where, $\sigma$ is attenuation coefficient and $z$ is the link distance.

\section{A. Pointing Error Model}

The fundamental reasons that introduces pointing error in optical signal is either due to misalignment of transceiver or vibrations of the buildings. Assuming, the Gaussian beam as beam waist radius $w_{z}$ reaches at distance $z$ received on photo-detector having a radius of $r$. The expression for power collected at $z=0$ is denoted by $A_{0}$. The pointing error random variable $H_{p}$ can be written as [8]

$$
H_{p}(\varsigma ; z) \approx A_{o} \exp \left(-\left(\frac{\varsigma \sqrt{2}}{\omega_{\text {zeq }}}\right)^{2}\right)
$$

The PDF of random variable $H_{p}$ is expressed as [30]

$$
f_{H_{p}}\left(H_{p}\right)=\frac{\varsigma^{2} H_{p} \varsigma^{2}-1}{A_{o}^{\varsigma^{2}}}, 0 \leq X \leq A_{o}
$$


where $A_{o}=[\operatorname{erf}(v)]^{2}$ is at radial displacement $z=0$ and $v=\sqrt{\frac{\pi}{2}} x \frac{a}{\omega_{z}}$. The parameter $\varsigma=\frac{\omega_{z e q}}{2 \sigma_{s}}$ is the ratio of equivalent beam radius $\omega_{z e q}$ and jitter standard deviation $\sigma_{s} . \$ \omega_{z e q}^{2}$ is the equivalent beam radius written as

$$
\omega_{z e q}^{2}=\frac{\omega_{z}^{2} \sqrt{\pi} \operatorname{erf}(v)}{2 v \exp \left(-v^{2}\right)}
$$

where $\omega_{z}=\sqrt{\omega_{o}^{2}\left(1+\varepsilon\left(\lambda L\left(\pi \omega_{o}^{2}\right)^{-2}\right)\right)}$ with $\omega_{0}$ radius of transmitted beam at $z=0, \varepsilon=2 \omega_{o}^{2} \rho_{o}^{-2}+$ 1 and the parameter $\rho_{o}=1.43\left(L k^{2} C_{n}^{2}\right)^{-\frac{3}{5}}$ is coherence length. Radial displacement $r=\sqrt{x^{2}+y^{2}}$ represents the displacement of optical beam in X-Y plane. Beckman distribution have two independent Gaussian random variables with non-zero means $\left(\mu_{x}, \mu_{y}\right)$ and variance $\left(\sigma_{x}^{2}, \sigma_{y}^{2}\right)$, the PDF of random variable $r$ can be modeled as Beckman distribution expressed as [31]

$$
f_{\zeta}(\varsigma)=\frac{\varsigma}{2 x \pi x \sigma_{x} x \sigma_{y}} x \int_{0}^{2 \pi} \exp \left(-\frac{\left(r \cos \theta-\mu_{x}\right)^{2}}{2 \sigma_{x}^{2}}-\frac{\left(r \sin \theta-\mu_{y}\right)^{2}}{2 \sigma_{y}^{2}}\right) d \theta
$$

\section{B. Rayleigh Pointing Error Model}

To develop Rayleigh pointing error model, substituting the displacement in $\mathrm{x}$-axis and $\mathrm{y}$-axis as zero mean $\mu_{x}=\mu_{y}=0$ and variances $\sigma_{s}^{2}=\sigma_{x}^{2}=\sigma_{y}^{2}$ in equation (38). The Rayleigh distribution in terms of radial displacement $\$$ lzeta $\$$ and jitter variance $\sigma_{s}^{2}$ can be written as [8]

$$
F_{\varsigma}(\varsigma)=\frac{\varsigma}{\sigma_{s}^{2}} \exp \left(-\frac{\varsigma^{2}}{2 \sigma_{s}^{2}}\right)
$$

Where the $\zeta$ is known as intensity of pointing error effect. 


\section{Rician Pointing Error Model}

Assuming, that radial displacement $\$ \mathbf{r} \$$ is non-zero mean $\left(\mu_{x} \neq \mu_{y}\right)$ and same variances $\left(\sigma_{s}^{2}=\right.$ $\sigma_{x}^{2}=\sigma_{y}^{2}$ ), where $\sigma_{s}^{2}$ is jitter variance then equation equation (38) becomes

$$
f_{\varsigma}(\varsigma)=\frac{\varsigma}{\sigma_{s}^{2}} \exp \left(-\frac{s^{2}+\varsigma^{2}}{2 \sigma_{s}^{2}}\right) \operatorname{Io}\left(\frac{\varsigma s}{\sigma_{s}^{2}}\right)
$$

where, $s=\sqrt{\mu_{x}^{2}+\mu_{y}^{2}}$ and $I_{0}($.$) is the modified Bessel function of first kind and zero order.$

Rearranging equation (35) and substituting the value of $r$ in equation (40), the PDF for Rician pointing error with non-zero bore-sight can be written as

$$
f_{H_{p}}\left(H_{p}\right)=\frac{\varsigma^{2} \exp \left(\frac{-s^{2}}{2 \sigma_{S}^{2}}\right)}{A_{o} \varsigma^{2}} H_{p} \varsigma^{2}-1 I_{o}\left(\frac{s}{\sqrt{2} \sigma_{s}^{2}} \sqrt{-\omega_{z e q}^{2} \ln \frac{H_{p}}{A_{o}}}\right)
$$

\section{Atmospheric Turbulence Model}

The random variable $\left(H_{a}\right)$ can be modeled as Log-Normal distribution. In FSO communication, Log-Normal is used to illustrate the weak atmospheric fading effect. The PDF of Log-Normal distribution is expressed as

$$
f_{H_{a}}\left(H_{a}\right)=\frac{1}{2 H_{a} \sqrt{2 \pi \sigma_{I}^{2}}} \exp \left(-\frac{\left(\ln \left(H_{a}\right)+2 \sigma_{I}^{2}\right)^{2}}{8 \sigma_{I}^{2}}\right)
$$


where, $\sigma_{I}^{2}=0.31 k^{\frac{7}{6}} C_{n}^{2} z^{\frac{11}{6}}$ is $\log$ amplitude variance, the relation of log-amplitude variance with Raytov variance is $\sigma_{I}^{2} \approx \frac{\sigma_{R}^{2}}{4}, \sigma_{R}^{2}$ is Raytov variance. $k=\frac{2 \pi}{\lambda}$ is the optical wave no. and $\lambda$ is the wavelength.

\section{E. Generalized Composite Channel Model}

The density function of equation (33) can be expressed as [8]

$$
f\left(H, w_{z}\right)=\int f_{H / H_{a}}\left(\frac{H}{H_{a}}\right) f_{H_{a}}\left(H_{a}\right) d H_{a}
$$

where, $f\left(H, w_{z}\right)$ is composite channel PDF with parameters $\$ H \$$ channel gain and $w_{z}$ is beam radius at distance $z, f_{H / H_{a}}$ is conditional that can be expressed as

$$
f_{H / H_{a}}\left(\frac{H}{H_{a}}\right)=\frac{1}{H_{a} H_{l}} f_{H_{p}} \frac{H}{H_{a} H_{l}}
$$

where, $H_{l}$ is deterministic quantity mentioned in equation (34). By substituting equation (39) and equation (41) in equation (44), the conditional probability becomes

$$
\begin{gathered}
f_{H / H_{a}}\left(\frac{H}{H_{a}}\right)=\frac{\varsigma^{2}}{A_{o}^{\varsigma^{2}} H_{a} H_{l}}\left(\frac{H}{H_{a} H_{l}}\right)^{\varsigma^{2}-1} \\
f_{H / H_{a}}\left(\frac{H}{H_{a}}\right)=\frac{\varsigma^{2} \exp \left(-\frac{s^{2}}{2 \sigma_{s}^{2}}\right)}{\left(A_{o} H_{a} H_{l}\right)^{\varsigma^{2}}} H^{\varsigma^{2}-1} \times I_{o}\left(\frac{s}{\sqrt{2} \sigma_{S}^{2}} \sqrt{-\omega_{z e q}^{2} \ln \frac{H_{p}}{A_{o}}}\right)
\end{gathered}
$$




\section{F. Log-Normal Channel with Rayleigh Pointing Error model}

Now, substituting equation (42) and equation (45) in equation (43) becomes

$$
f_{H}\left(H, \omega_{z}\right)=\frac{\varsigma^{2} H^{\varsigma^{2}-1}}{2\left(A_{o} H_{l}\right)^{\varsigma^{2}}} \operatorname{erfc}\left(\frac{\ln \left(\frac{H}{A_{o} H_{l}}\right)+\mu}{\sqrt{8} \sigma_{x}}\right) \exp (a)
$$

Where $a=2 \sigma_{x}^{2} \varsigma^{2}\left(1+\varsigma^{2}\right)$. Now, we will derive the moment generating function for equation

(47). Assuming that $x=\frac{\ln \left(\frac{H}{A_{o} H_{l}}\right)+\mu}{\sqrt{8} \sigma_{x}}$ and $H$ can be written as

$$
H=A_{o} H_{l} \exp \left(x \sqrt{8} \sigma_{x}-\mu\right)
$$

the equation (47) can be written as

$$
f_{H}\left(H, \omega_{z}\right)=\frac{\varsigma^{2} H^{\varsigma^{2}-1}}{2\left(A_{o} H_{l}\right) \varsigma^{2}} \operatorname{erfc}(x) \exp (a)
$$

the approximation for $\operatorname{erfc}($.$) can be written as [32]$

$$
\operatorname{erfc}(x)=\left(a_{1} t+a_{2} t^{2}+a_{3} t^{3}\right) \exp \left(-x^{2}\right), t=\frac{1}{1+p x}, x \geq 0
$$

where $p=0.47047, a_{1}=0.3480242, a_{2}=-0.0958798$ and $a_{3}=0.7478556$. Now, letting $f(t)=a_{1} t+a_{2} t^{2}+a_{3} t^{3}$ and substituting equation (50) in equation (49) 


$$
f_{H}\left(H, \omega_{z}\right)=\frac{\varsigma^{2} H^{\varsigma^{2}-1}}{2\left(A_{o} H_{l}\right)^{2}} f(t) \exp \left(-x^{2}\right) \exp (a)
$$

now substituting equation (51)in equation (9), it becomes

$$
I(\beta, \varphi)=2 \int_{0}^{\infty} Q(\beta, \varphi) \frac{\varsigma^{2} H^{\varsigma^{2}-1}}{2\left(A_{o} H_{l}\right)^{\varsigma^{2}}} f(t) \exp \left(-x^{2}\right) \exp (a) d x
$$

now applying Craig's formula in equation (52) using equation (14), new equation becomes

$$
I(\beta, \varphi)=\frac{2}{\pi} \int_{0}^{\infty} \int_{0}^{\varphi} \exp \left(-\frac{y^{2}}{2 \sin ^{2} \theta}\right) \frac{\varsigma^{2} H^{\varsigma^{2}-1}}{2\left(A_{o} H_{l}\right)^{2}} f(t) \exp \left(-x^{2}\right) \exp (a) d \theta d x
$$

Now substituting $y=\sqrt{2 D \gamma(I)}$, putting equation (48), $\gamma(I)=\frac{H^{2}}{2 \sigma_{I}^{2}}=\frac{A_{o}^{2}}{2 \sigma_{I}^{2}}\left(H_{l} \exp \left(x \sqrt{8} \sigma_{x}-\mu\right)\right)^{2}$ and $\gamma(I)=k_{o}\left(H_{l} \exp \left(x \sqrt{8} \sigma_{x}-\mu\right)\right)^{2}$. Now, substituting the value of $y^{2}$, applying Gauss Hermite Quadrature in equation (53) and simplification give rise to

$$
\begin{gathered}
I(\beta, \varphi)=\frac{\varsigma^{2} \exp (a)}{\pi A_{o} H_{l}} \sum_{i=0}^{\infty} \omega_{i} f(t)\left(\exp \left(x \sqrt{8} \sigma_{x}-\mu\right)\right)^{\varsigma^{2}-1} \\
\times \int_{0}^{\varphi} \exp \left(-\frac{2 D k_{o}\left(H_{l} \exp \left(x \sqrt{8} \sigma_{x}-\mu\right)\right)^{2}}{2 \sin ^{2} \theta}\right) d \theta
\end{gathered}
$$

now closing the Craig's form, the new equation becomes

$$
I(\beta, \varphi)=\frac{\varsigma^{2} \exp (a)}{\pi A_{o} H_{l}} \sum_{i=0}^{\infty} \omega_{i}\left(a_{1} t+a_{2} t^{2}+a_{3} t^{3}\right)\left(\exp \left(x \sqrt{8} \sigma_{x}-\mu\right)\right)^{\varsigma^{2}-1}
$$




$$
\times Q\left(\sqrt{2 D k_{o}}\left(H_{l} \exp \left(x \sqrt{8} \sigma_{x}-\mu\right)\right)\right)
$$

The above closed form mathematical expression is $M G F$ to evaluate the ASER for Log-Normal with Rayleigh P.E model.

\section{G. Log-Normal Channel with Rician Pointing Error model}

Now, substituting equation (42) and equation (46) in equation (43) becomes

$$
f_{H}\left(H, w_{z}\right)=\frac{\varsigma^{2} \exp \left(x_{a}\right) H^{\varsigma^{2}-1}}{2\left(A_{o} H_{l}\right)^{\varsigma^{2}}} \operatorname{erfc}\left(\frac{\ln \left(\frac{H}{A_{o} H_{l}}\right)+x_{b}}{x_{c}}\right)
$$

where, $x_{a}=2 \sigma_{x}^{2} \varsigma^{4}+2 \sigma_{x}^{2} \varsigma^{2}+\frac{s^{2}}{\sigma_{s}^{2}}, x_{b}=4 \sigma_{x}^{2} \varsigma^{2}+2 \sigma_{x}^{2}+\frac{6 s^{2}}{w_{z e q}^{2}}$, and $x_{c}=\sqrt{8\left(\frac{4 s^{2} \sigma_{s}^{2}}{w_{z e q}^{4}}+\sigma_{x}^{2}\right)}$.

Now, deriving moment generating function for equation (56), assuming that $x=\frac{\ln \left(\frac{H}{A_{o} H_{l}}\right)+x_{b}}{x_{c}}$ and $H$ can be written as

$$
H=A_{o} H_{l} \exp \left(x x_{c}-x_{b}\right)
$$

now putting the value of $x$, applying $\operatorname{erfc}(x)$ numerical approximation and substituting in equation (9) it becomes

$$
I(\beta, \varphi)=\frac{\varsigma^{2} \exp \left(x_{a}\right)}{\left(A_{o} H_{l}\right) \varsigma^{2}} \int_{0}^{\infty} Q(\beta, \varphi) H^{\varsigma^{2}-1} f(t) \exp \left(-x^{2}\right) d x
$$


Now applying Craig's approximation, Gauss Hermite Quadrature approximation and substituting $y^{2}=\left(2 D k_{o}\right)\left(H_{l} \exp \left(x \sqrt{8} \sigma_{x}-\mu\right)\right)^{2}$, value of $H$ and after simplifying equation (58) becomes

$$
\begin{aligned}
& I(\beta, \varphi)=\frac{\varsigma^{2} \exp \left(x_{a}\right)}{\pi A_{o} H_{l}} \sum_{i=0}^{\infty} \omega_{i} f(t)\left(\exp \left(x x_{c}-x_{b}\right)\right)^{\varsigma^{2}-1} \\
& \times \int_{0}^{\varphi} \exp \left(-\frac{2 D k_{o}\left(H_{l} \exp \left(x x_{c}-x_{b}\right)\right)^{2}}{2 \sin ^{2} \theta}\right) d \theta
\end{aligned}
$$

closing Q-function according to Craig's approximation, the new expression becomes

$$
\begin{aligned}
& I(\beta, \varphi)=\frac{\varsigma^{2} \exp (a)}{\pi A_{o} H_{l}} \sum_{i=0}^{\infty} \omega_{i}\left(a_{1} t+a_{2} t^{2}+a_{3} t^{3}\right)\left(\exp \left(x x_{c}-x_{b}\right)\right)^{\varsigma^{2}-1} \\
& Q\left(\sqrt{2 D k_{o}}\left(H_{l} \exp \left(x x_{c}-x_{b}\right)\right)\right)
\end{aligned}
$$

The above closed form mathematical expression is $M G F$ to evaluate the ASER for Log-Normal with Rician P.E model.

\section{ASER Analysis}

In this section, we will derive ASER expression of TQAM that is corrupted by turbulent channel in the presence of P.E with zero and non-zero boresight. The work shown below has not been found in the literature previously. The authors' have ensured that the analytical expression presented below is easily computable on any computational software like Mathematica or MATLAB. The ASER of TQAM in FSO can be calculated as

$$
P_{e}=\int_{0}^{\infty} P_{s e}(\beta) f_{r}(r) d r
$$


where, $P_{s e}(\beta)$ is symbol error probability $(S E P)$ and $f_{r}(r)$ is PDF of SNR $(r)$ received. The SEP of regular $M_{\text {ary }}$ TQAM is given as by [22]

$$
\begin{aligned}
& P_{s e}(\beta)=6\left(1-\frac{4}{\sqrt{3 M}}+\frac{1}{3 M}\right) Q(\beta)-15\left(1-\frac{7}{3 \sqrt{M}}+\frac{6}{5 M}\right) Q^{2}(\beta) \\
& +20\left(1-\frac{61}{20 \sqrt{M}}+\frac{11}{5 M}\right) Q^{3}(\beta)-15\left(1-\frac{53}{15 \sqrt{M}}+\frac{46}{15 M}\right) Q^{4}(\beta) \\
& +6\left(1-\frac{23}{6 \sqrt{M}}+\frac{11}{3 M}\right) Q^{5}(\beta)-\left(1-\frac{4}{\sqrt{M}}+\frac{4}{M}\right) Q^{6}(\beta)
\end{aligned}
$$

where the $\beta=\sqrt{2 D r}$ is normalized minimum distance between neighboring symbols and $D$ is defined as $D=\frac{12}{7 M-4}$. In equation $(61) Q(\beta)=2 Q\left(\beta, \frac{\pi}{2}\right)$ and $Q^{2}(\beta)=2 Q\left(\beta, \frac{\pi}{4}\right)$ can be computed as using Craig's approximation

$$
Q(\beta, \varphi)=\frac{1}{2 \pi} \int_{0}^{\varphi} \exp \left(-\frac{\beta^{2}}{2 \sin ^{2} \theta}\right) d \theta
$$

where in [61], the higher power of Q-function can be computed as $Q^{3}(\beta)=Q(\beta) Q^{2}(\beta)$, $Q^{4}(\beta)=Q^{2}(\beta) Q^{2}(\beta), Q^{5}(\beta)=Q^{2}(\beta) Q^{3}(\beta)$ and $Q^{6}(\beta)=Q^{3}(\beta) Q^{3}(\beta)$. To evaluate the ASEP of Log-Normal shadowing, Rayleigh, Rician, Log-Normal with Rayleigh P.E and Log-Normal with Rician P.E fading channels using equation (18), equation (26), equation (32), equation (55) and equation (60) respectively can be substituted in the following expression, which is given as 


$$
\begin{aligned}
& P_{\text {fading }}=6\left(1-\frac{4}{\sqrt{3 M}}+\frac{1}{3 M}\right) I(D, \varphi)-15\left(1-\frac{7}{3 \sqrt{M}}+\frac{6}{5 M}\right) I^{2}(D, \varphi) \\
& +20\left(1-\frac{61}{20 \sqrt{M}}+\frac{11}{5 M}\right) I^{3}(D, \varphi)-15\left(1-\frac{53}{15 \sqrt{M}}+\frac{46}{15 M}\right) I^{4}(D, \varphi) \\
& +6\left(1-\frac{23}{6 \sqrt{M}}+\frac{11}{3 M}\right) I^{5}(D, \varphi)-\left(1-\frac{4}{\sqrt{M}}+\frac{4}{M}\right) I^{6}(D, \varphi)
\end{aligned}
$$

To evaluate ASEP of Log-Normal Rayleigh and Rician P.E can also be computed by substituting equation (47) and equation (56) in equation (64) by using following expression, which can be written as

$$
I(D, \varphi)=\int_{0}^{\infty} Q^{i}(\sqrt{2 D r}, \varphi) f_{H}\left(H, w_{z}\right) d H
$$

Where $i=1,2, \ldots, 6$.

Table 1 System parameters and constants.

\section{Numerical results and discussion}

In this section, we will evaluate and compare the already derived analytical expression for MTQAM in Log-Normal shadowing, Rician fading, Rayleigh fading, Log-normal with Rayleigh

pointing error (with boresight displacement), and Log-Normal with Rician pointing error(without 
boresight displacement) respectively. The Log-Normal shadowing, Rician fading, and Rayleigh fading channels depicts weak, moderate, and strong turbulence having a scintillation index less than 0.5 , less than 0.75 , and great than 1 respectively. The performance of M-TQAM in afore mentioned channels are evaluated as symbol error rate against $\operatorname{SNR}\left(E_{S} / N_{0}\right)$ and average received irradiance $\left(I_{0}\right)$. The symbol error performance of M-TQAM against average irradiance $\left(I_{0}\right)$ has been observed against two values of SNR i.e. $0 d B$ and $20 d B$. Throughout the analysis, other parameters such as Responstivity and modulation index is considered unity.

Figure 2 The 16-TQAM ASEP performance against average received irradiance $I_{0}$ in LogNormal Shadowing at $S N R=0 \mathrm{db}$ and $20 \mathrm{~dB}$.

Figure 3 The 16-TQAM ASEP performance versus average received irradiance $I_{0}$ in Rician fading channel at Rician $K_{\text {factor }}=4 d B, S N R=0 d b$ and $20 d B$.

Figure 4 The 16-TQAM ASEP performance versus average received irradiance $I_{0}$ in Rayleigh fading channel at $S N R=0 \mathrm{db}$ and $20 \mathrm{~dB}$.

In Figure 2, 3 and 4 the symbol error performance of 16-TQAM in Log-Normal shadowing, Rician fading and Rayleigh fading is observed against average irradiance $I_{0}$ respectively. From the figures, it can be seen that Log-Normal exhibits the least requirements of irradiance $I_{0}$ and 16-TQAM performs better in Log-Normal as having low SEP. Log-Normal shadowing displays 
weak turbulence and has least scintillation index among the other fading channels. It has been observed that by increasing the SNR per symbol the requirement of irradiance received decreased and for the same values of received irradiance at $(0 d B)$, the SEP has been improved. Now, as we compare the Rician and Rayleigh Fading channels, it has been observed that received irradiance demand is lesser in Rician fading. The performance of the Rician fading channel can be improved either by adjusting the Rician k-factor or increasing SNR per symbol $\left(E_{s} / N_{0}\right)$. In Figure 3 and 4 the performance of 16-TQAM in the related fading channels have been improved by increasing the SNR per symbol from $(0 \mathrm{~dB})$ to $(20 \mathrm{~dB})$ (see Table 2 and 3). The symbol error rate of $10^{-3}$ using 16-TQAM in Log-Normal, Rayleigh and Rician requires irradiance intensity of $-71,48$ and 37 respectively.

Table 2 ASER vs. $I_{0}$ with $E_{s} / N_{0} 0 d B$ and $20 d B$ over Rayleigh Fading Channel.

Table 3 ASER vs. $I_{0}$ with $E_{S} / N_{0} 0 d B$ and $20 d B$ over Rician Fading Channel.

Table 4 ASER vs. $I_{0}$ with $E_{S} / N_{0} 0 d B$ and $20 d B$ over Rician Fading Channel.

When the transmitted optical signal travels through the Free-space reaches the receiver, it gets tempered. That received signal is recovered compared with the transmitted set of symbols. The ratio of received symbols in error to total transmitted is calculated. This ratio gives an estimated probability of symbols in error for that value of SNR. In Figure 5 symbol error rate of 16-TQAM in Log-Normal, Rician, and Rayleigh fading has been plotted as a function of SNR, in Table 4 a 
SEP vs. SNR comparison of 16-TQAM on afore mentioned channels has been presented. The SEP of 16-TQAM in Log-Normal shadowing. The SEP of 16-TQAM in Log-Normal shadowing decreases significantly as the SNR increases. The 16-TQAM in Rician fading channel performs better than Rayleigh fading channel due to the presence of specular (LOS) component. Furthermore, these theoretical results have been validated through simulation results. These simulation results follow the theoretical results closely and show a close agreement.

Figure 5 The 16-TQAM ASEP performance versus average SNR $\gamma$ in Log-Normal, Rician $\left(K_{\text {factor }}=16 d B\right)$ and Rayleigh fading channels.

Table 5 ASER vs. $I_{0}$ with $E_{s} / N_{0} 0 d B$ and $20 d B$ over Rician Fading Channel.

Figure 6 and 7 illustrates ASER performance against transmitter beam radius as a running parameter for different values of weather effects $C_{n}^{2}$ and pointing error standard deviation $\sigma_{s}$ with Rayleigh as pointing error and Rician pointing error respectively. As the weather effects $C_{n}^{2}$ and jitter's standard deviation $\sigma_{s}$ increases, the ASER also increases. Moreover, as the value of transmitter beam radius approaches as closer to $w_{0} \approx 0.022 m$ the ASER decreases and eventually at $w_{0} \approx 0.022 \mathrm{~m}$ ASER is minimum. The ASER performance for given conditions is therefore optimized and $w_{0} \approx 0.022 m$ is an optimal value. In Figure 6, due to Rayleigh pointing error model is employed the ASER value is smaller than Rician pointing error model at $w_{0} \approx$ 
$0.022 \mathrm{~m}$ due to the presence of boresight displacement $s=0.02$ in Rician pointing model (Figure 7). The difference in the values of ASER can be seen in Table 5 at $w_{0} \approx 0.022 \mathrm{~m}$.

Figure 6 The ASEP performance against optical beamwidth $w_{0}$ over various values of turbulent conditions $C_{n}^{2}$ and P.E standard deviation $\sigma_{s}$ in Log-Normal with Rayleigh P.E model employing receiver aperture radius $r=0.052 \mathrm{~m}$, optical link displacement $z=1000 \mathrm{~m}$ and average $\mathrm{SNR}=30 \mathrm{~dB}$.

Figure 7 The ASEP performance against optical beamwidth $w_{0}$ over various values of turbulent conditions $C_{n}^{2}$ and P.E standard deviation $\sigma_{s}$ in Log-Normal with Rician P.E model employing receiver aperture radius $r=0.052 m$, optical link displacement $z=1000 \mathrm{~m}$ and average SNR $=30 \mathrm{~d} B$.

In Figure 8 and 9, the ASER is evaluated against the transmitter beam radius for Rayleigh pointing error model and Rician pointing error model respectively, for different values of receiver aperture radius and weather conditions. The effect of aperture radius on the performance of optical communication is significance. From the figures, it can be seen that as the receiver's aperture size increases the ASER gets improved. The aperture radius $r$ at $0.042 \mathrm{~m}$ and $0.052 \mathrm{~m}$ gives significantly poor ASER performance than $r$ at $0.062 \mathrm{~m}$. Moreover, at an optical beam radius \$w_0 \approx0.022 m\$ the ASER gives the minimum values, that is the optimal value of optical beam radius in all weather conditions.

Figure 8 The ASEP performance against optical beamwidth $w_{0}$ over various values of turbulent conditions $C_{n}^{2}$ and receiver aperture radius $r$ in Log-Normal with Rayleigh P.E model employing P.E standard deviation $\sigma_{s}=0.07 \mathrm{~m}$, optical link displacement $z=1000 \mathrm{~m}$ and average $S N R=30 \mathrm{~dB}$. 
Figure 9 The ASEP performance against optical beamwidth $w_{0}$ over various values of turbulent conditions $C_{n}^{2}$ and receiver aperture radius $r$ in Log-Normal with Rician P.E model employing P.E standard deviation $\sigma_{s}=0.07 \mathrm{~m}$, optical link displacement $z=1000 \mathrm{~m}$ and average $S N R=30 d B$.

Figure 10 and 11 depicts the combined effect of different weather conditions i.e. weak $(1 \times$ $\left.10^{-15}\right)$, moderate $\left(9 \times 10^{-15}\right)$ and strong $\left(3 \times 10^{-14}\right)$, optical beam width having values $\left(w_{0}=0.02 \mathrm{~m}\right),\left(w_{0}=0.022 \mathrm{~m}\right)$ and $\left(w_{0}=0.024 \mathrm{~m}\right)$ and jitter's standard deviation as a running parameter on ASER with rayleigh and the Rician pointing error model respectively. Clearly, it can be seen that as the value of jitter's standard deviation increases, ASER also increases. The composite effect of weather conditions and an increase in jitter's standard deviation make ASER increase and system performance becomes poor. It is worth observing that the effect of P.E is more visible when the atmospheric turbulence is strong. Practically, the effect of P.E standard deviation cannot be minimized. By selecting the right width of the optical beamwidth the performance of the system can be optimized. But P.E effect cannot be reduced by only adjusting the optical beamwidth. In Figure 11, due to the employing of the Rician pointing error model, the ASER is slightly higher than the Rayleigh P.E model.

Figure 10 The ASEP performance against P.E displacement standard deviation $\sigma_{s}$ over various values of turbulent conditions $C_{n}^{2}$ and optical beamwidth $w_{0}$ in Log-Normal with Rayleigh P.E model employing receiver aperture radius $r=0.052 \mathrm{~m}$, optical link displacement $z=1000 \mathrm{~m}$ and average $S N R=30 \mathrm{~dB}$. 
Figure 11 The ASEP performance against P.E displacement standard deviation $\sigma_{s}$ over various values of turbulent conditions $C_{n}^{2}$ and optical beamwidth $w_{0}$ in Log-Normal with Rician P.E model employing receiver aperture radius $r=0.052 \mathrm{~m}$, optical link displacement $z=1000 \mathrm{~m}$ and average $S N R=30 d B$.

In Figure 12 and 13, the combined effect of weak, moderate and strong turbulence, the effect of receiver aperture radius having values of $r=0.042,0.052$ and 0.062 and as function of jitters standard deviation the ASER has been plotted at $w_{-} 0=0.022$. These figures are plotted for rayleigh and the Rician pointing error model. It can be seen in the figures that by increasing the radius of the receiver aperture the combine effect of weather conditions and P.E standard deviation on ASER can be reduced significantly. ASER decreases with the increase in radius of aperture. By selecting the right size of aperture radius and by employing optimal width of optical beam the effect of jitters standard deviation $\sigma_{s}$ can be minimized.

Figure 12 The ASEP performance against P.E displacement standard deviation $\sigma_{s}$ over various values of turbulent conditions $C_{n}^{2}$ and receiver aperture radius $r$ in Log-Normal with Rayleigh P.E model employing optical beamwidth $w_{0} \approx 0.022 \mathrm{~m}$, optical link displacement $z=1000 \mathrm{~m}$ and average $S N R=30 d B$.

Figure 13 The ASEP performance against P.E displacement standard deviation $\sigma_{s}$ over various values of turbulent conditions $C_{n}^{2}$ and receiver aperture radius $r$ in Log-Normal with Rician P.E model employing optical beamwidth $w_{0} \approx 0.022 \mathrm{~m}$, optical link displacement $z=1000 \mathrm{~m}$ and average $S N R=30 d B$. 
Figure 14 and 15, emphasis more on the selection of receiver's aperture radius to enhance the performance of optical communication link. In Figure 15, the Rayleigh pointing error model with Log-Normal has been plotted for different values of optical beam width $w_{-} 0=0.014,0.018,0.022$ and 0.026 , weak atmospheric turbulence $C_{n}^{2}=1 \times 10^{-15}$, linkspan $z=1000 m$, P.E standard deviation $\sigma_{s}=0.1$ and $S N R=30 \mathrm{~dB}$. The span of optical link is $1000 m$. in Figure 15, the Rician P.E model is employed for the same parameters as mentioned before. As we know, due to the presence of boresight displacement $s=0.01$ in Rician pointing error model, it gives slightly higher values of ASER. In Figure 14 and 15, the falling curve with optimal beam requires small aperture and it performs better than others in terms of ASER. Also, it can be observed that by increasing the size of receivers aperture the ASER performance gets improved.

Figure 14 The ASEP performance against receiver aperture radius $r$ over various values of optical beamwidth $w_{0}$ in Log-Normal with Rayleigh P.E model employing P.E displacement standard deviation $\sigma_{S}=0.07 \mathrm{~m}$, optical link displacement $z=1000 \mathrm{~m}$ and average $S N R=$ $30 d B$.

Figure 15 The ASEP performance against receiver aperture radius $r$ over various values of optical beamwidth $w_{0}$ in Log-Normal with Rician P.E model employing P.E displacement standard deviation $\sigma_{S}=0.07 \mathrm{~m}$, optical link displacement $z=1000 \mathrm{~m}$ and average $S N R=$ $30 d B$. 
Figure 16 and 17, the rayleigh, and Rician P.E models have been plotted respectively. ASER of these models has been observed against receiver aperture radius for various values of P.E standard deviation $\sigma_{s}=0.05, \sigma_{s}=0.07, \sigma_{s}=0.1 \$$ in meters, weather condition $C_{n}^{2}=1 \times$ $10^{-15}, S N R=30 d B$ and link length $z=1000 \mathrm{~m}$. we can not control the jitter standard deviation $\sigma_{s}$. however, the effect of P.E standard deviation can be reduced by using the right size of the receiver aperture. As the aperture radius size increases the ASER decreases drastically.

Figure 16 The ASEP performance against receiver aperture radius $r$ over various values of P.E displacement standard deviation $\sigma_{s}$ in Log-Normal with Rayleigh P.E model employing optical beamwidth $w_{0}=0.022 \mathrm{~m}$, optical link displacement $z=1000 \mathrm{~m}$ and average $S N R=30 \mathrm{~dB}$.

Figure 17 The ASEP performance against receiver aperture radius $r$ over various values of P.E displacement standard deviation $\sigma_{s}$ in Log-Normal with Rician P.E model employing optical beamwidth $w_{0}=0.022 \mathrm{~m}$, optical link displacement $z=1000 \mathrm{~m}$ and average $S N R=30 \mathrm{~dB}$.

In Figure 18 and 19, the ASER of Log-Normal pointing error models have been plotted for various values optical beam width $w_{0}=0.02,0.022$ and 0.024 , in strong weather conditions and P.E $\sigma_{s}=0.07$ against Signal to noise ratio (SNR). It can be observed from the figures that as the SNR increases, the ASER decreases Sharply. The waterfall curve with optical beamwidth of $w_{0}=0.024$ performs better than $w_{0}=0.02$ due to a wider beam and it is well collected by the 
aperture of the receiver. Furthermore, the optical beam width $w_{0}=0.022$ performs better among the others.

Figure 18 The ASEP performance against average SNR over various values of optical beamwidth $w_{0}$ in Log-Normal with Rayleigh P.E model employing P.E displacement standard deviation $\sigma_{s}=0.07 \mathrm{~m}$, optical link displacement $z=1000 \mathrm{~m}$ and receiver aperture radius $r=0.052 m$.

Figure 19 The ASEP performance against average SNR over various values of optical beamwidth $w_{0}$ in Log-Normal with Rician P.E model employing P.E displacement standard deviation $\sigma_{s}=0.07 \mathrm{~m}$, optical link displacement $z=1000 \mathrm{~m}$ and receiver aperture radius $r=0.052 m$.

Figure 20, demonstrate the ASER performance of an optical communication system in strong turbulence condition $1 \times 10^{-15}$, receivers aperture radius $r=0.062$, P.E standard deviation $\sigma_{S}=0.07$, optical beam width $w_{0}=0.022$ and SNR as a running parameter for two different multiplicities of regular TQAM $(M=16,64)$. It is been seen that as the average electrical SNR increases the ASER decreases. 
Figure 20 The M-TQAM $(M=16,64)$ ASEP performance against average SNR in Log-Normal with P.E model incorporating zero boresight $(s=0)$ and nonzero boresight $(s=0.05)$ employing P.E displacement standard deviation $\sigma_{s}=0.07 m$, optical beamwidth $w_{0} \approx 0.022 m$, turbulent condition $1 \times 10^{-15} \mathrm{~m}^{-2 / 3}$,optical link displacement $z=1000 \mathrm{~m}$ and receiver aperture radius $\mathrm{r}=0.052 \mathrm{~m}$.

\section{Conclusion}

In this article, we have investigated the performance of SC-TQAM over Log-Normal, Rayleigh and Rician fading channels. We have also investigated Log-Normal distribution with weak, moderate and strong atmospheric turbulence employed P.E effect with zero and non-zero boresight. The moment generating function of Log-Normal with Rayleigh and Rician P.E models has also been derived. The expression for ASER is derived and analyzed against the LASER average received irradiance, optical beam width, P.E standard deviation, receiver side aperture, and SNR. The selection of these parameters is critically important to achieve better performance in weak to strong turbulence conditions accordingly. Moreover, ASER performance is observed against SNR and Monte Carlo simulation is performed that validates the theoretical results, at higher values of SNR simulation and theoretical results show a close agreement.

\section{List of Abbreviations}

$\mathrm{FSO}=$ Free Space Optical

QAM = Quadrature Amplitude Modulation

TQAM = Triangular Quadrature Amplitude Modulation

ASER = Average Symbol Error Rate

SQAM = Square Quadrature Amplitude Modulation 
SC-TQAM = Subcarrier Triangular Quadrature Amplitude Modulation

P.E $=$ Pointing Errors

EMI $=$ Electromagnetic Interference

LOS $=$ Line of Sight

OOK $=$ On Off Keying

PPM = Pulse Position Modulation

MPSK $=$ Multiple Phase Shift Keying

MPAM $=$ Multiple Pulse Amplitude Modulation

$\mathrm{IM}=$ Intensity Modulation

BPSK $=$ Binary Phase Shift Keying

XQAM $=$ Cross Quadrature Amplitude Modulation

RQAM = Rectangular Quadrature Amplitude Modulation

HCSS $=$ Honey Comb signal set

TSSS $=$ Triangular Shape Signal Set

$\mathrm{RF}=$ Radio Frequency

$\mathrm{PD}=$ Photo Detector

AWGN = Additive White Gaussian Noise

SER $=$ Symbol Error Rate

$\mathrm{MGF}=$ moments generating function

SNR = signal-to-noise ratio

$\mathrm{pdf}=$ probability density function

$\mathrm{dB}=$ deciBel

\section{Availability of Data and Materials}


Signalling points generated for the simulation purposes were based on the structure of TQAM.

\section{Competing Interests}

The authors declare that they have no competing interests.

\section{Funding}

There is no funding for the research work presented in this article.

\section{Author's Contributions}

MN wrote the article and did the initial research work. FH presented the idea of how TQAM can be tested in Free Space Optics. Research was done under supervision of SAS.

QUK and MZ did their part dealing with moment generating functions.

\section{Acknowledgements}

Authors are thankful to head of department, Department of Electrical Engineering, College of Electrical and Mechanical Engineering, National University of Sciences and Technology, Dr Fahad Mumtaz Malik for providing a pleasant and well-suited environment for research.

\section{References}

1. A. K. Majumdar and J. C. Ricklin, Free-space laser communications: principles and advances, vol. 2 (Springer Science \& Business Media, 2010).

2. A. Mansour, R. Mesleh, and M. Abaza, "New challenges in wireless and free space optical communications,” Opt. lasers engineering 89, 95-108 (2017).

3. H. Singh and A. S. Sappal, "Analytic and simulative comparison of turbulent fso system with different modulation techniques," Opt. \& Laser Technol. 114, 49-59 (2019). 
4. H. Singh and A. S. Sappal, "Moment-based approach for statistical and simulative analysis of turbulent atmospheric channels in fso communication," IEEE Access 7, 11296-11317 (2019).

5. W. O. Popoola, Z. Ghassemlooy, and J. Allen, "Performance of subcarrier modulated free-space optical communications," in 8th Annual post graduate symposium on the convergence of telecommunications, networking and broadcasting (PGNet), (2007), pp. $75-80$.

6. N. Sharma and P. Garg, "Cross-qam signaling in free space optical communication systems with generalized pointing errors," in 2017 IEEE 86th Vehicular Technology Conference (VTC-Fall), (IEEE, 2017), pp.1-5.

7. H. D. Trung, A. T. Pham et al., "Pointing error effects on performance of free-space optical communication systems using sc-qam signals over atmospheric turbulence channels, AEU-International J. Electron. Commun. 68, 869-876 (2014).

8. A. A. Farid and S. Hranilovic, "Outage capacity optimization for freespace optical links with pointing errors," J. Light. technology 25, 1702-1710 (2007).

9. W. Gappmair, S. Hranilovic, and E. Leitgeb, "Ook performance for terrestrial fso links in turbulent atmosphere with pointing errors modeled by hoyt distributions," IEEE communications letters 15, 875-877 (2011). S. Rajbhandari, Z. Ghassemlooy, and M. Angelova, "A study of discrete

10. wavelet transform based denoising to reduce the effect of artificial light interferences for indoor optical wireless communication," in $20107^{\text {th }}$ International Symposium on Communication Systems, Networks \&Digital Signal Processing (CSNDSP 2010), (IEEE, 2010), pp. 610-614. 
11. S. G. Wilson, M. Brandt-Pearce, Q. Cao, and J. H. Leveque, "Freespace optical mimo transmission with q-ary ppm,” IEEE Transactions on Commun. 53, 1402-1412 (2005).

12. H. Yao, X. Ni, C. Chen, B. Li, X. Zhang, Y. Liu, S. Tong, Z. Liu, and H. Jiang, "Performance of m-pam fso communication systems in atmospheric turbulence based on apd detector,” Opt. express 26, 23819-23830 (2018).

13. H. Yao, X. Ni, C. Chen, B. Li, X. Feng, X. Liu, Z. Liu, S. Tong, and H. Jiang, "Performance analysis of mpsk fso communication based on the balanced detector in a fiber-coupling system,” IEEE Access 7, 84197-84208 (2019).

14. J. Smith, "Odd-bit quadrature amplitude-shift keying," IEEE Transactions on Commun. 23, 385-389 (1975).

15. F. H. Qureshi, Q. U. Khan, S. A. Sheikh, and M. Zeeshan, "Symbol error probability performance of rectangular qam with mrc reception over generalized $a-m$ fading channels," IEICE TRANSACTIONS on Fundamentals Electron. Commun. Comput. Sci. 101, 577-584 (2018).

16. M. Simon and J. Smith, "Hexagonal multiple phase-and-amplitude-shift keyed signal sets," IEEE Transactions on Commun. 21, 1108-1115 (1973).

17. S. Oshita, T. Yamazato, and S. Kondo, "Performance evaluation of modified qam and triangular shaped signal set," in Fourth IEEE Region 10 International Conference TENCON, (IEEE, 1989), pp. 158-161.

18. S.-J. Park, "Triangular quadrature amplitude modulation," IEEE communications letters 11, 292-294 (2007).

19. S.-J. Park, "Performance analysis of triangular quadrature amplitude modulation in awgn channel,” IEEE Commun. Lett. 16, 765-768 (2012). 
20. K.-K. Cho, J.-Y. Lee, and D.-W. Yoon, "Performance analysis of generalized triangular qam,” The J. Korean Inst. Commun. Inf. Sci. 35, 885-888 (2010).

21. T. T. Duy and H. Y. Kong, "A simple approximation for the symbol error rate of triangular quadrature amplitude modulation," IEICE transactions on communications 93, $753-756$ (2010).

22. F. H. Qureshi, Q. U. Khan, and S. A. Sheikh, "Sep performance of triangular qam with sc and gsc spatial diversity over rayleigh channels," in 2016 IEEE/ACIS 15th International Conference on Computer and Information Science (ICIS), (IEEE, 2016), pp. 1-5.

23. F. Haider Qureshi, S. Amin Sheikh, Q. Umar Khan, and F. Mumtaz Malik, "Sep performance of triangular qam with mrc spatial diversity over fading channels, qureshi et al,” EURASIP J. on Wirel. Commun. Netw. 5 (2016).

24. M. Viswanathan, "Simulation of digital communication systems using matlab," Mathuranathan Viswanathan at Smashwords (2013).

25. M. SIMONS and M. ALOUINI, "Digital communication over fading channels: A unified approach to performance analysis," (2000).

26. J. W. Goodman, Statistical optics (John Wiley \& Sons, 2015).

27. J. W. Craig, "A new, simple and exact result for calculating the probability of error for two-dimensional signal constellations," MILCOM 91 - Conf. record pp. 571-575 vol.2 (1991).

28. M. K. Simon, "A simpler form of the craig representation for the two dimensional joint gaussian q-function,” IEEE Commun. Lett. 6, 49-51 (2002).

29. J. M. Cioffi, "Ee379c advanced digital communications," Course reader (1998). 
30. A. Jurado-Navas, J. M. Garrido-Balsells, J. F. Paris, M. Castillo- Vázquez, and A. PuertaNotario, "Impact of pointing errors on the performance of generalized atmospheric optical channels," Opt. express 20, 12550-12562 (2012).

31. P. Beckmann and A. Spizzichino, "The scattering of electromagnetic waves from rough surfaces,” Norwood, MA, Artech House, Inc., 1987, 511 p. (1987).

32. M. Abramowitz and I. A. Stegun, Handbook of mathematical functionswith formulas, graphs, and mathematical tables, vol. 55 (US Government printing office, 1948).

\section{Legends for Specific Figures with Figure number and title are mentioned below:}

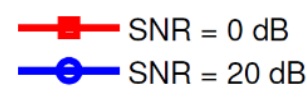

Figure 2 The 16-TQAM ASEP performance against average received irradiance $I_{0}$ in LogNormal Shadowing at $S N R=0 \mathrm{db}$ and $20 \mathrm{~dB}$.

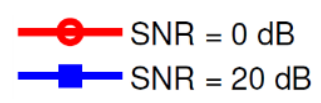

Figure 3 The 16-TQAM ASEP performance against average received irradiance $I_{0}$ in Rician fading channel at $S N R=0 \mathrm{db}$ and $20 \mathrm{~dB}$. 
$\begin{aligned} \longrightarrow \text { SNR } & =0 \mathrm{~dB} \\ -\mathrm{SNR} & =20 \mathrm{~dB}\end{aligned}$

Figure 4 The 16-TQAM ASEP performance against average received irradiance $I_{0}$ in Rayleigh fading channel at $S N R=0 \mathrm{db}$ and $20 \mathrm{~dB}$.

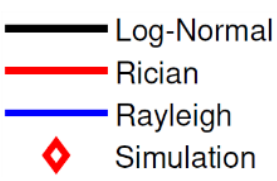

Figure 5 The 16-TQAM ASEP performance versus average SNR $\gamma$ in Log-Normal, Rician $\left(K_{\text {factor }}=16 d B\right)$ and Rayleigh fading channels.

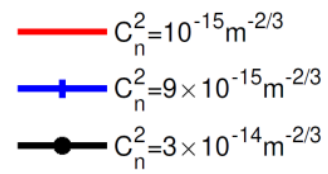

Figure 6 The ASEP performance against optical beamwidth $w_{0}$ over various values of turbulent conditions $C_{n}^{2}$ and P.E standard deviation $\sigma_{s}$ in Log-Normal with Rayleigh P.E model employing receiver aperture radius $r=0.052 m$, optical link displacement $z=1000 m$ and average $\mathrm{SNR}=30 \mathrm{~dB}$. 


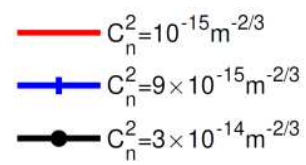

Figure 7 The ASEP performance against optical beamwidth $w_{0}$ over various values of turbulent conditions $C_{n}^{2}$ and P.E standard deviation $\sigma_{s}$ in Log-Normal with Rician P.E model employing receiver aperture radius $r=0.052 m$, optical link displacement $z=1000 m$ and average SNR $=30 \mathrm{~d} B$.

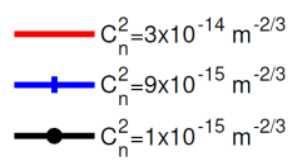

Figure 8 The ASEP performance against optical beamwidth $w_{0}$ over various values of turbulent conditions $C_{n}^{2}$ and receiver aperture radius $r$ in Log-Normal with Rayleigh P.E model employing P.E standard deviation $\sigma_{s}=0.07 \mathrm{~m}$, optical link displacement $z=1000 \mathrm{~m}$ and average $S N R=30 d B$.

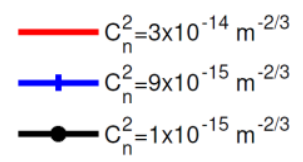

Figure 9 The ASEP performance against optical beamwidth $w_{0}$ over various values of turbulent conditions $C_{n}^{2}$ and receiver aperture radius $r$ in Log-Normal with Rician P.E model employing P.E standard deviation $\sigma_{s}=0.07 \mathrm{~m}$, optical link displacement $z=1000 \mathrm{~m}$ and average $S N R=30 d B$. 


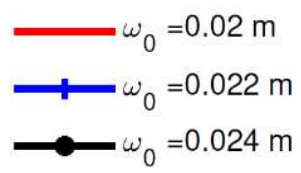

Figure 10 The ASEP performance against P.E displacement standard deviation $\sigma_{s}$ over various values of turbulent conditions $C_{n}^{2}$ and optical beamwidth $w_{0}$ in Log-Normal with Rayleigh P.E model employing receiver aperture radius $r=0.052 \mathrm{~m}$, optical link displacement $z=1000 \mathrm{~m}$ and average $S N R=30 \mathrm{~dB}$.

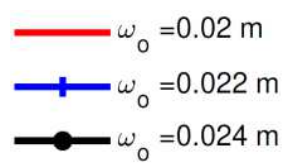

Figure 11 The ASEP performance against P.E displacement standard deviation $\sigma_{s}$ over various values of turbulent conditions $C_{n}^{2}$ and optical beamwidth $w_{0}$ in Log-Normal with Rician P.E model employing receiver aperture radius $r=0.052 \mathrm{~m}$, optical link displacement $z=1000 \mathrm{~m}$ and average $S N R=30 \mathrm{~dB}$.

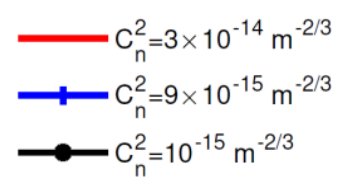

Figure 12 The ASEP performance against P.E displacement standard deviation $\sigma_{s}$ over various values of turbulent conditions $C_{n}^{2}$ and receiver aperture radius $r$ in Log-Normal with Rayleigh P.E model employing optical beamwidth $w_{0} \approx 0.022 \mathrm{~m}$, optical link displacement $z=1000 \mathrm{~m}$ and average $S N R=30 \mathrm{~dB}$. 


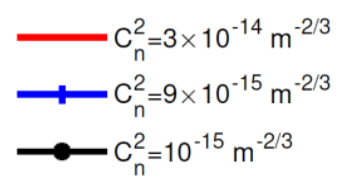

Figure 13 The ASEP performance against P.E displacement standard deviation $\sigma_{s}$ over various values of turbulent conditions $C_{n}^{2}$ and receiver aperture radius $r$ in Log-Normal with Rician P.E model employing optical beamwidth $w_{0} \approx 0.022 \mathrm{~m}$, optical link displacement $z=1000 \mathrm{~m}$ and average $S N R=30 \mathrm{~dB}$.

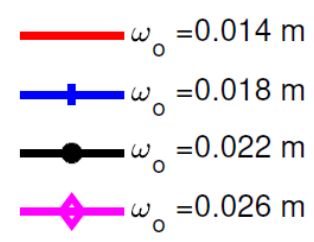

Figure 14 The ASEP performance against receiver aperture radius $r$ over various values of optical beamwidth $w_{0}$ in Log-Normal with Rayleigh P.E model employing P.E displacement standard deviation $\sigma_{S}=0.07 \mathrm{~m}$, optical link displacement $z=1000 \mathrm{~m}$ and average $S N R=$ $30 d B$.

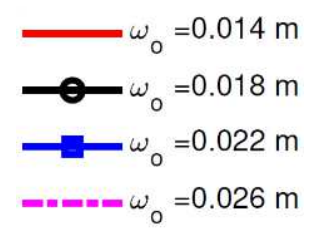

Figure 15 The ASEP performance against receiver aperture radius $r$ over various values of optical beamwidth $w_{0}$ in Log-Normal with Rician P.E model employing P.E displacement 
standard deviation $\sigma_{S}=0.07 \mathrm{~m}$, optical link displacement $z=1000 \mathrm{~m}$ and average $S N R=$ $30 d B$.

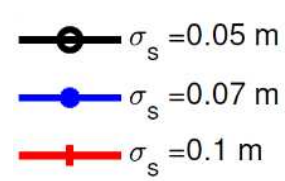

Figure 16 The ASEP performance against receiver aperture radius $r$ over various values of P.E displacement standard deviation $\sigma_{s}$ in Log-Normal with Rayleigh P.E model employing optical beamwidth $w_{0}=0.022 \mathrm{~m}$, optical link displacement $z=1000 \mathrm{~m}$ and average $S N R=30 \mathrm{~dB}$.

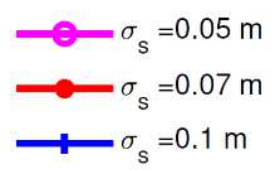

Figure 17 The ASEP performance against receiver aperture radius $r$ over various values of P.E displacement standard deviation $\sigma_{s}$ in Log-Normal with Rician P.E model employing optical beamwidth $w_{0}=0.022 \mathrm{~m}$, optical link displacement $z=1000 \mathrm{~m}$ and average $S N R=30 \mathrm{~dB}$.

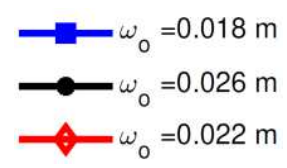

Figure 18 The ASEP performance against average SNR over various values of optical beamwidth $w_{0}$ in Log-Normal with Rayleigh P.E model employing P.E displacement standard deviation $\sigma_{s}=0.07 m$, optical link displacement $z=1000 \mathrm{~m}$ and receiver aperture radius $r=0.052 m$. 


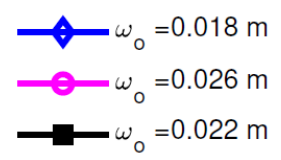

Figure 19 The ASEP performance against average SNR over various values of optical beamwidth $w_{0}$ in Log-Normal with Rician P.E model employing P.E displacement standard deviation $\sigma_{s}=0.07 \mathrm{~m}$, optical link displacement $z=1000 \mathrm{~m}$ and receiver aperture radius $r=0.052 m$.

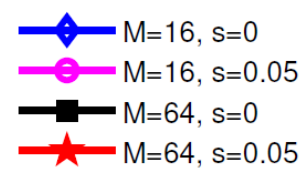

Figure 20 The M-TQAM $(M=16,64)$ ASEP performance against average SNR in Log-Normal with P.E model incorporating zero boresight $(s=0)$ and nonzero boresight $(s=0.05)$ employing P.E displacement standard deviation $\sigma_{s}=0.07 m$, optical beamwidth $w_{0} \approx 0.022 m$, turbulent condition $1 \times 10^{-15} \mathrm{~m}^{-2 / 3}$,optical link displacement $z=1000 \mathrm{~m}$ and receiver aperture radius $\mathrm{r}=0.052 \mathrm{~m}$. 
Figures

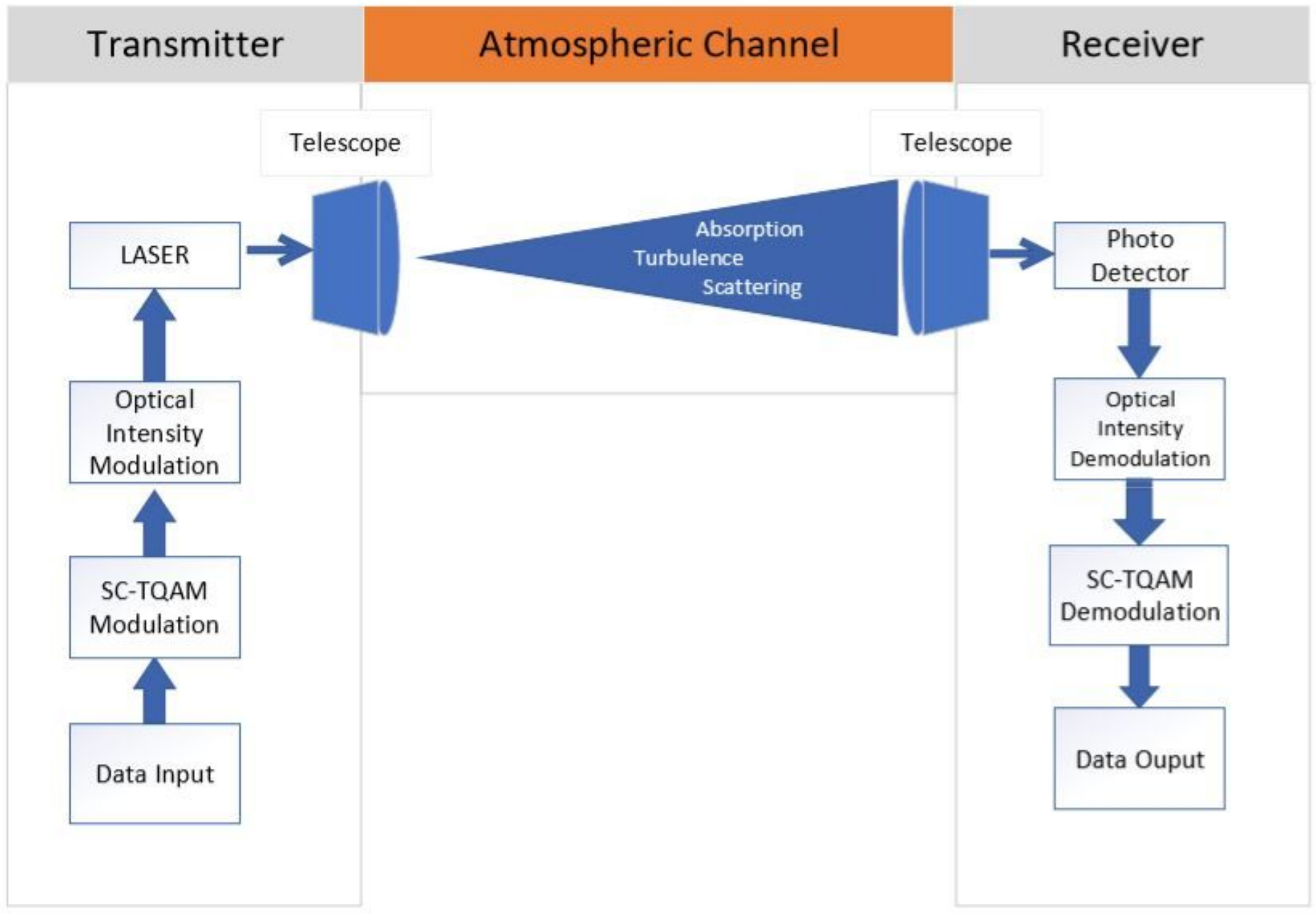

Figure 1

Basic block diagram of FSO communication system 


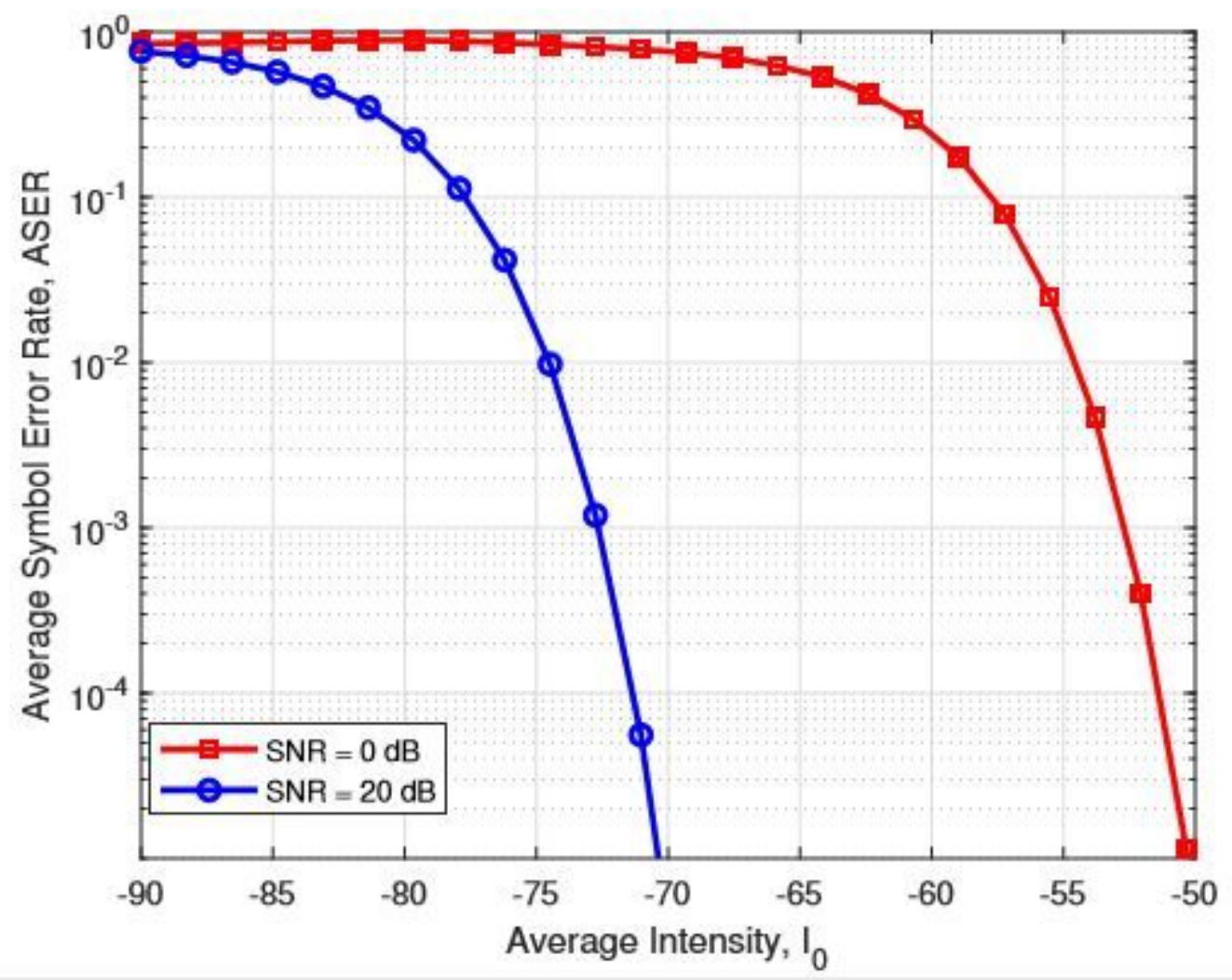

Figure 2

The 16-TQAM ASEP performance against average received irradiance I_0 in Log-Normal Shadowing at $S N R=0 \mathrm{db}$ and $20 \mathrm{~dB}$.

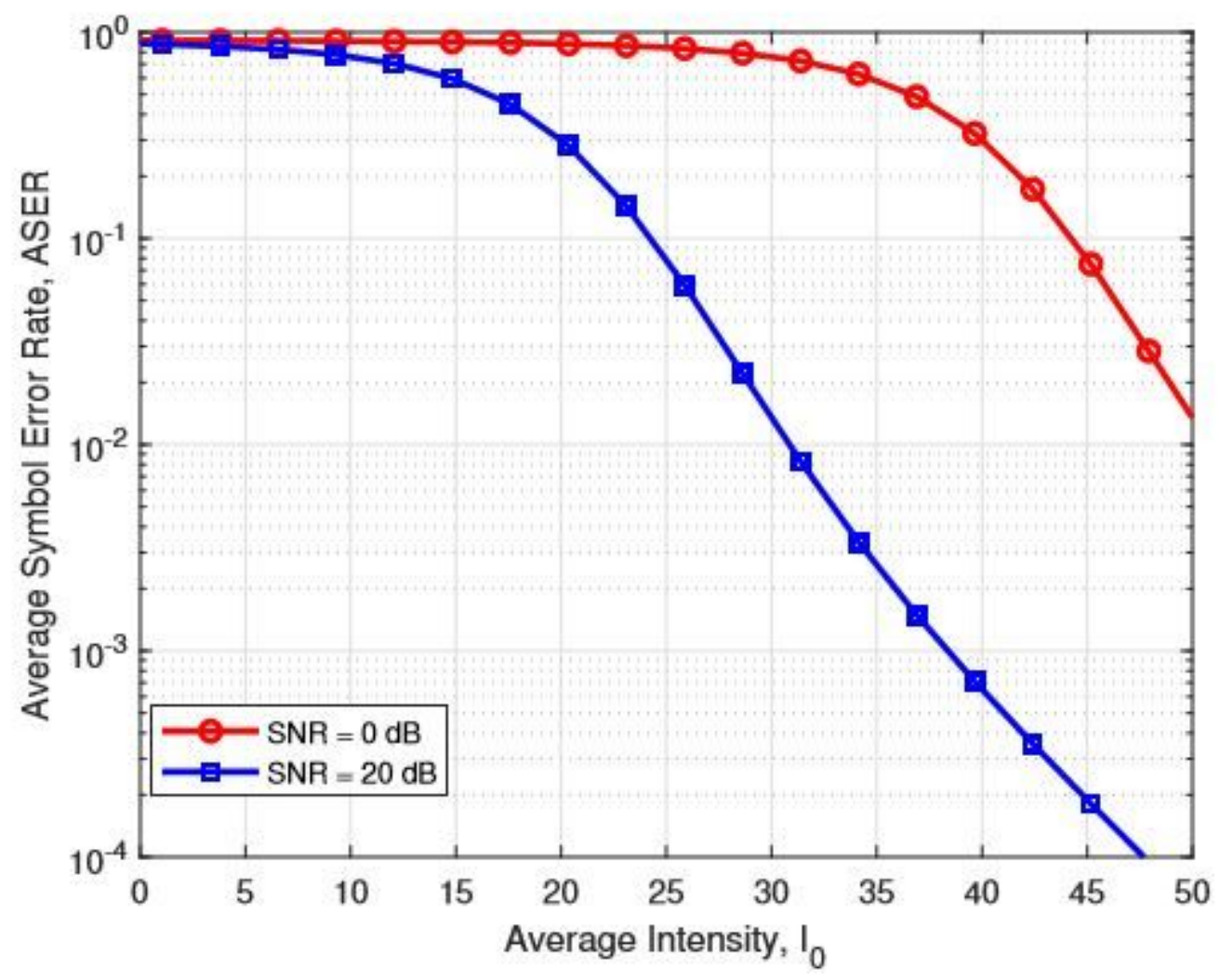


Figure 3

The 16-TQAM ASEP performance versus average received irradiance I_0 in Rician fading channel at Rician K_factor $=4 \mathrm{~dB}, \mathrm{SNR}=0 \mathrm{db}$ and $20 \mathrm{~dB}$.

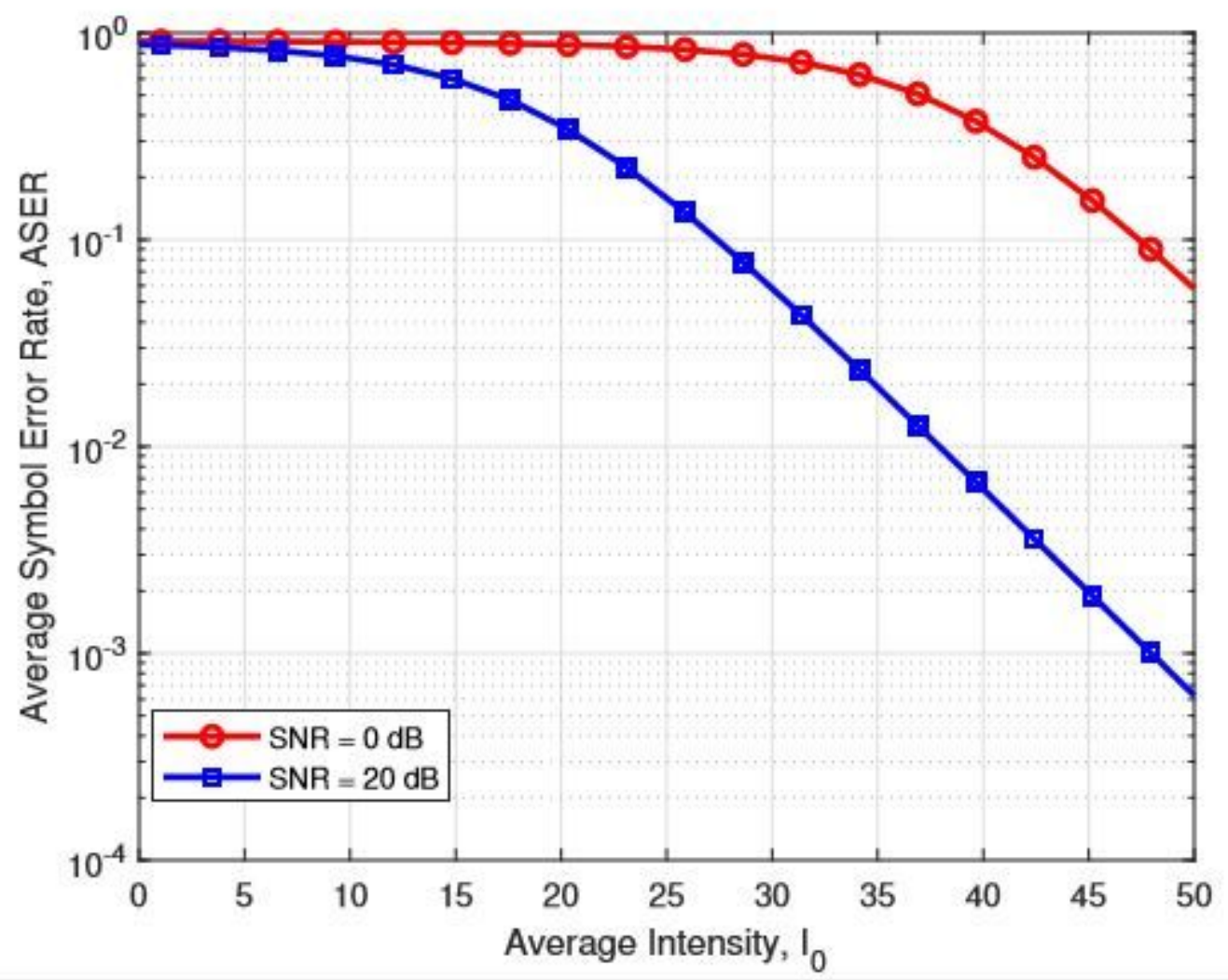

Figure 4

The 16-TQAM ASEP performance versus average received irradiance I_0 in Rayleigh fading channel at $S N R=0 \mathrm{db}$ and $20 \mathrm{~dB}$. 


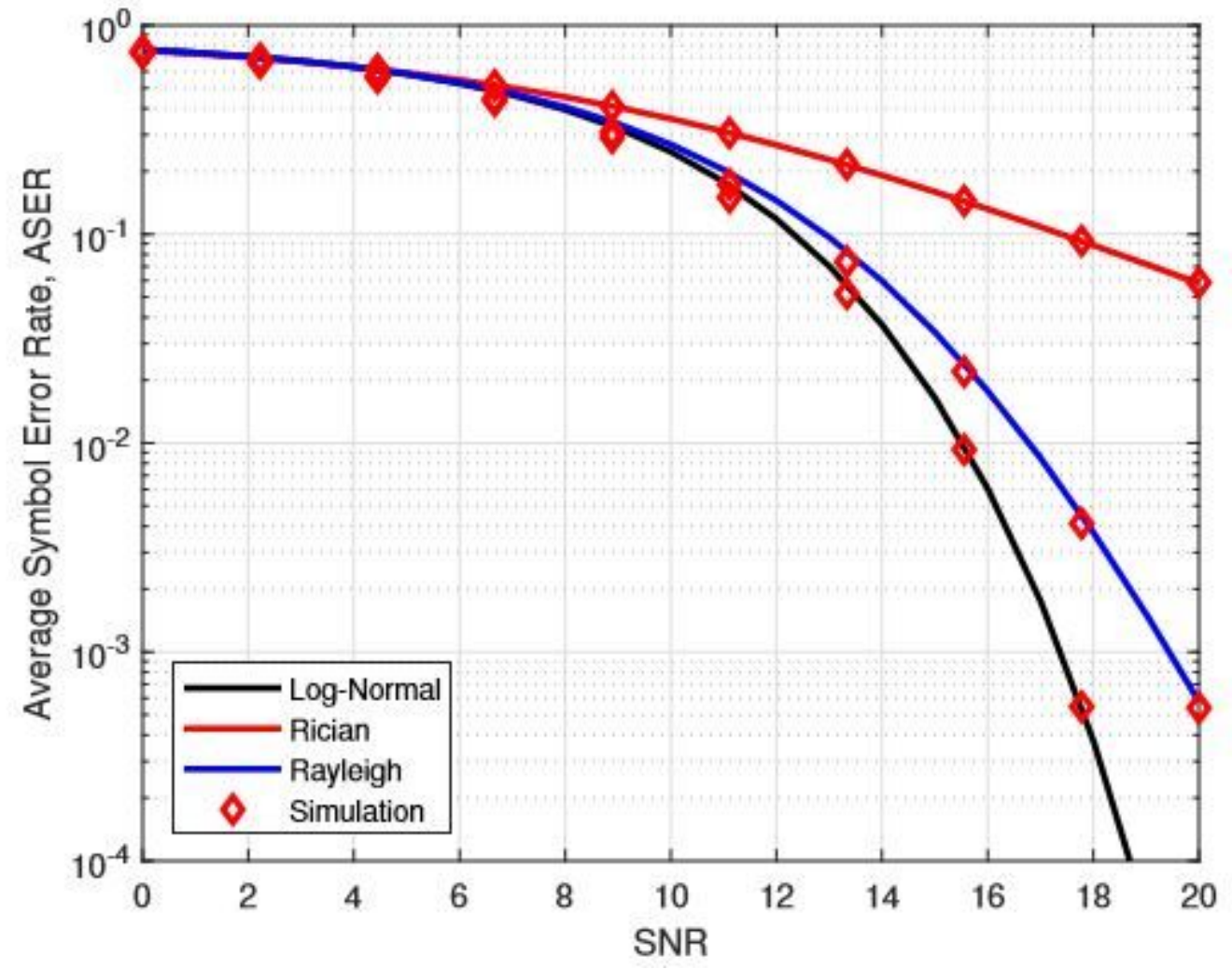

Figure 5

The 16-TQAM ASEP performance versus average SNR Y in Log-Normal, Rician (K_factor=16dB) and Rayleigh fading channels.

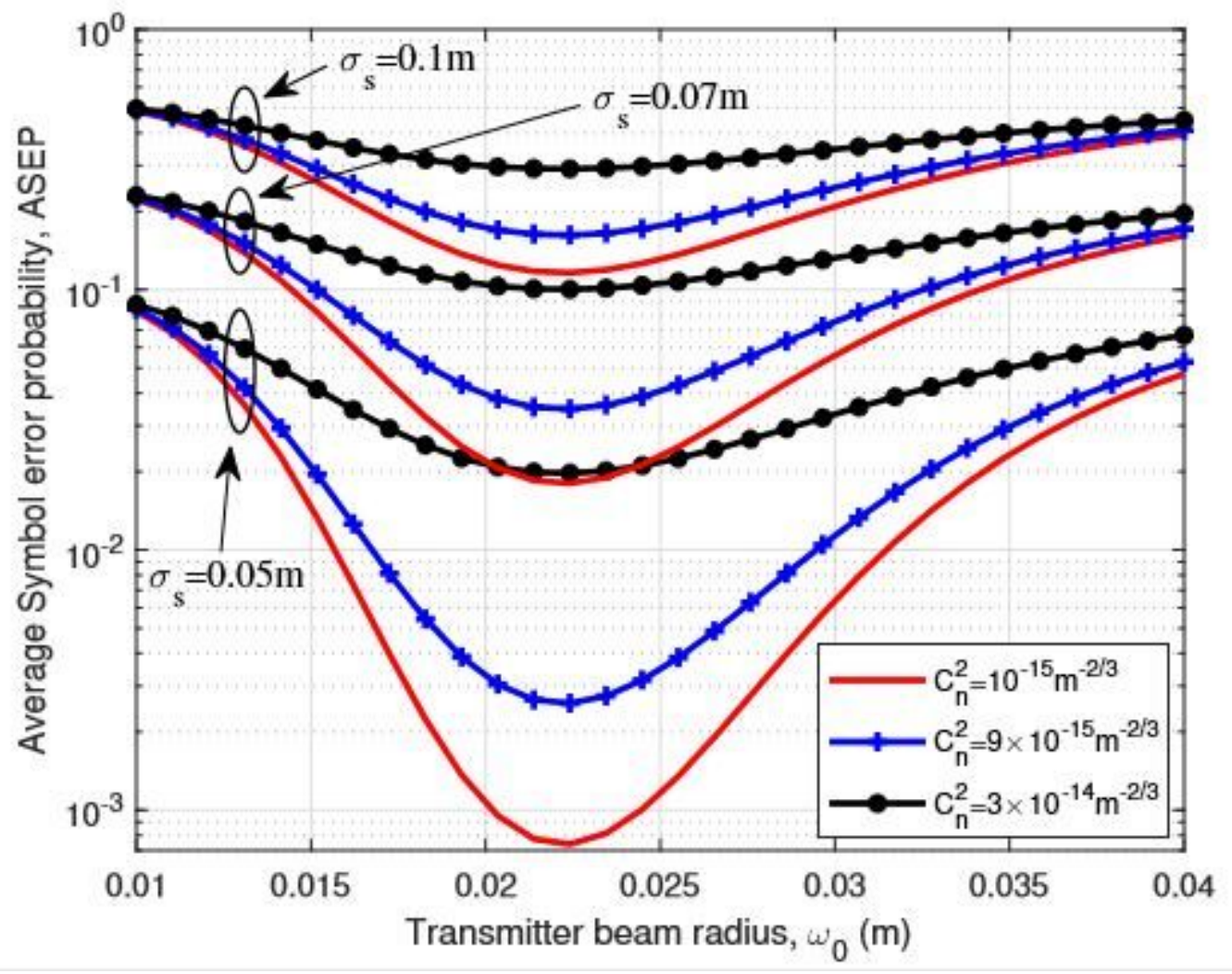


Figure 6

The ASEP performance against optical beamwidth $\mathrm{w}_{-} 0$ over various values of turbulent conditions C_n^2 and P.E standard deviation $\sigma \_s$ in Log-Normal with Rayleigh P.E model employing receiver aperture radius $r=0.052 \mathrm{~m}$, optical link displacement $\mathrm{z}=1000 \mathrm{~m}$ and average $S N R=30 \mathrm{~dB}$.

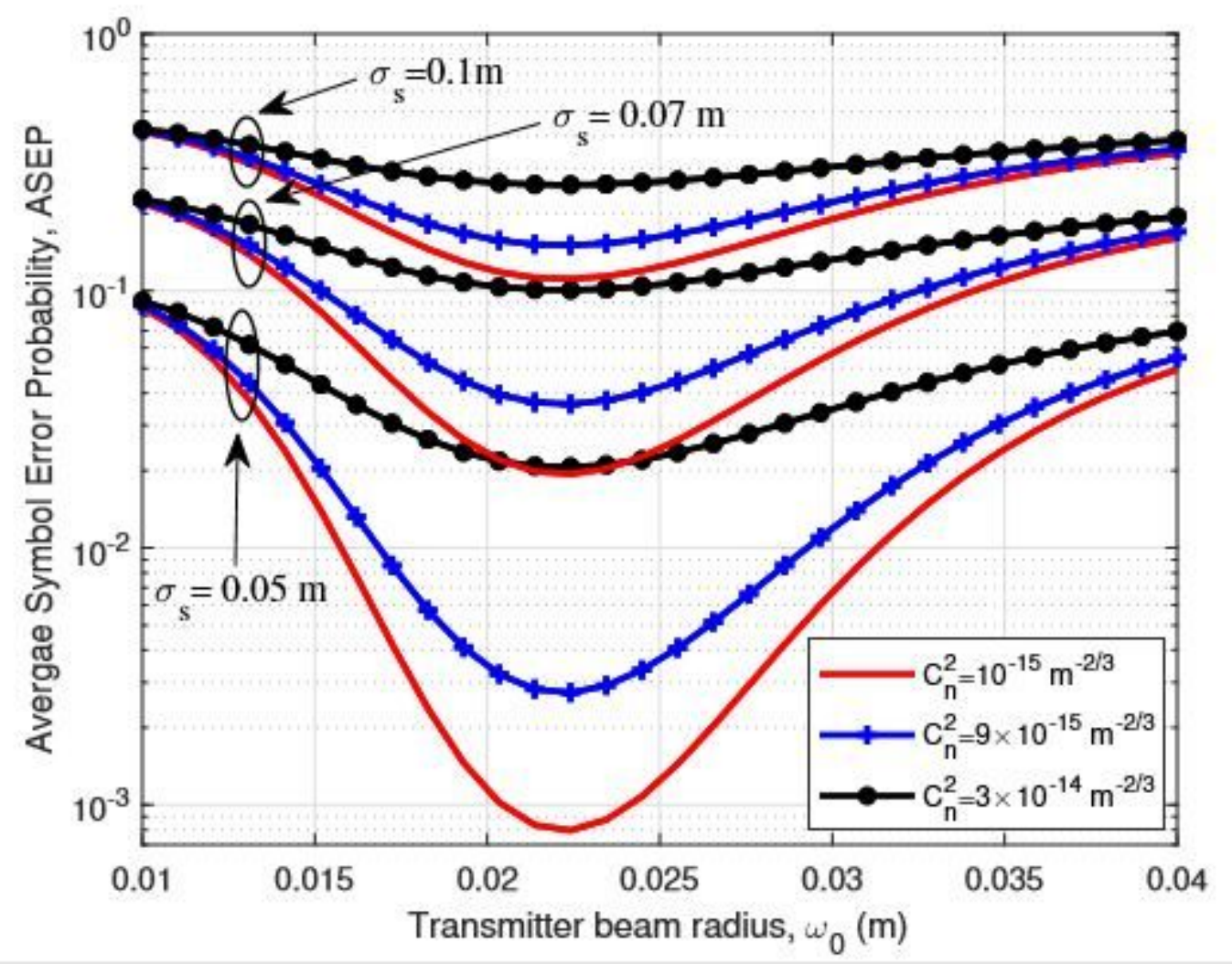

Figure 7

The ASEP performance against optical beamwidth $w_{-} 0$ over various values of turbulent conditions $C_{-} n^{\wedge} 2$ and P.E standard deviation $\sigma \_s$ in Log-Normal with Rician P.E model employing receiver aperture radius $r=0.052 \mathrm{~m}$, optical link displacement $\mathrm{z}=1000 \mathrm{~m}$ and average $\mathrm{SNR}=30 \mathrm{~dB}$. 


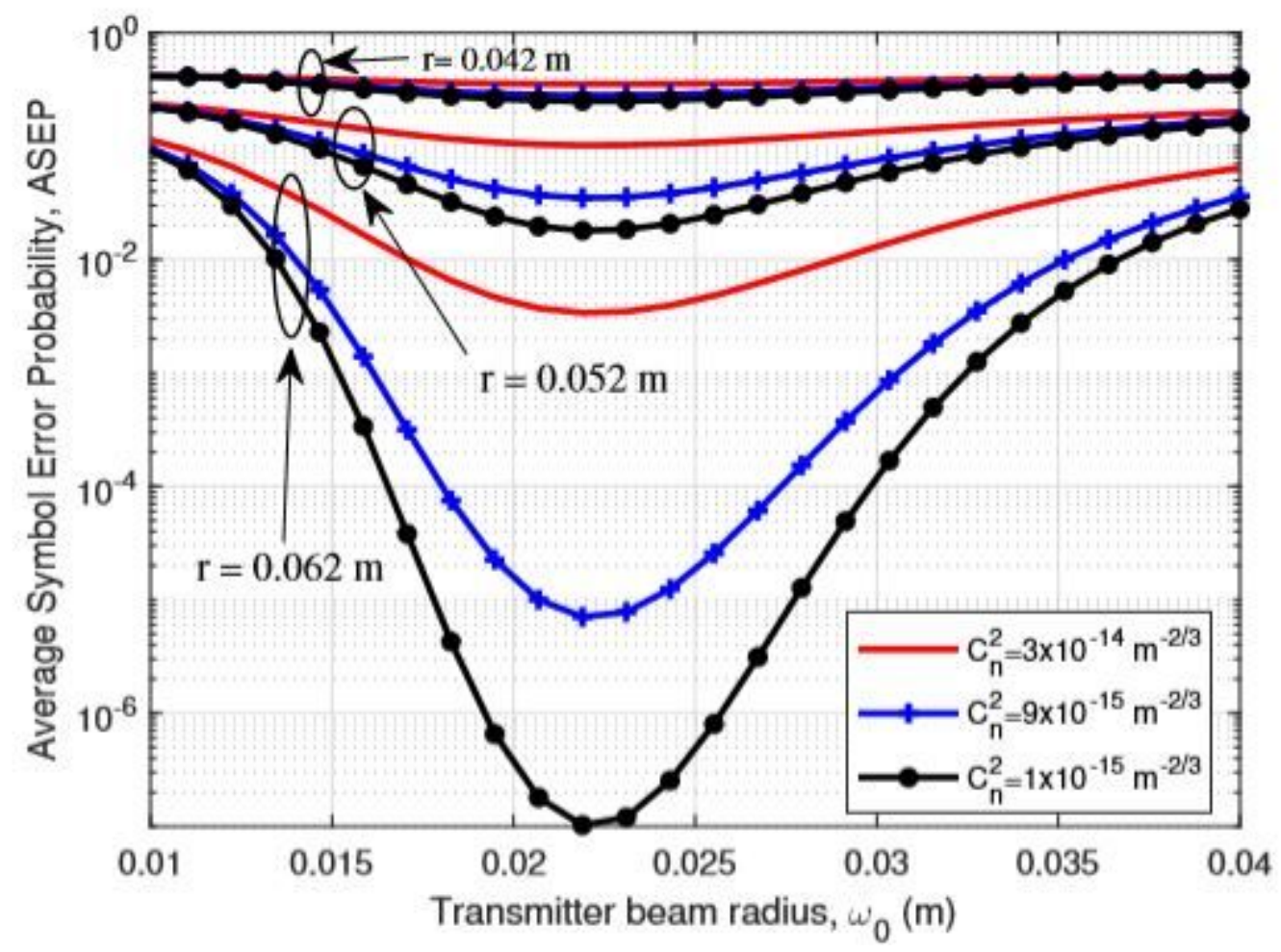

Figure 8

The ASEP performance against optical beamwidth w_0 over various values of turbulent conditions C_n^2 and receiver aperture radius $r$ in Log-Normal with Rayleigh P.E model employing P.E standard deviation $\sigma \_s=0.07 \mathrm{~m}$, optical link displacement $\mathrm{z}=1000 \mathrm{~m}$ and average $S N R=30 \mathrm{~dB}$.

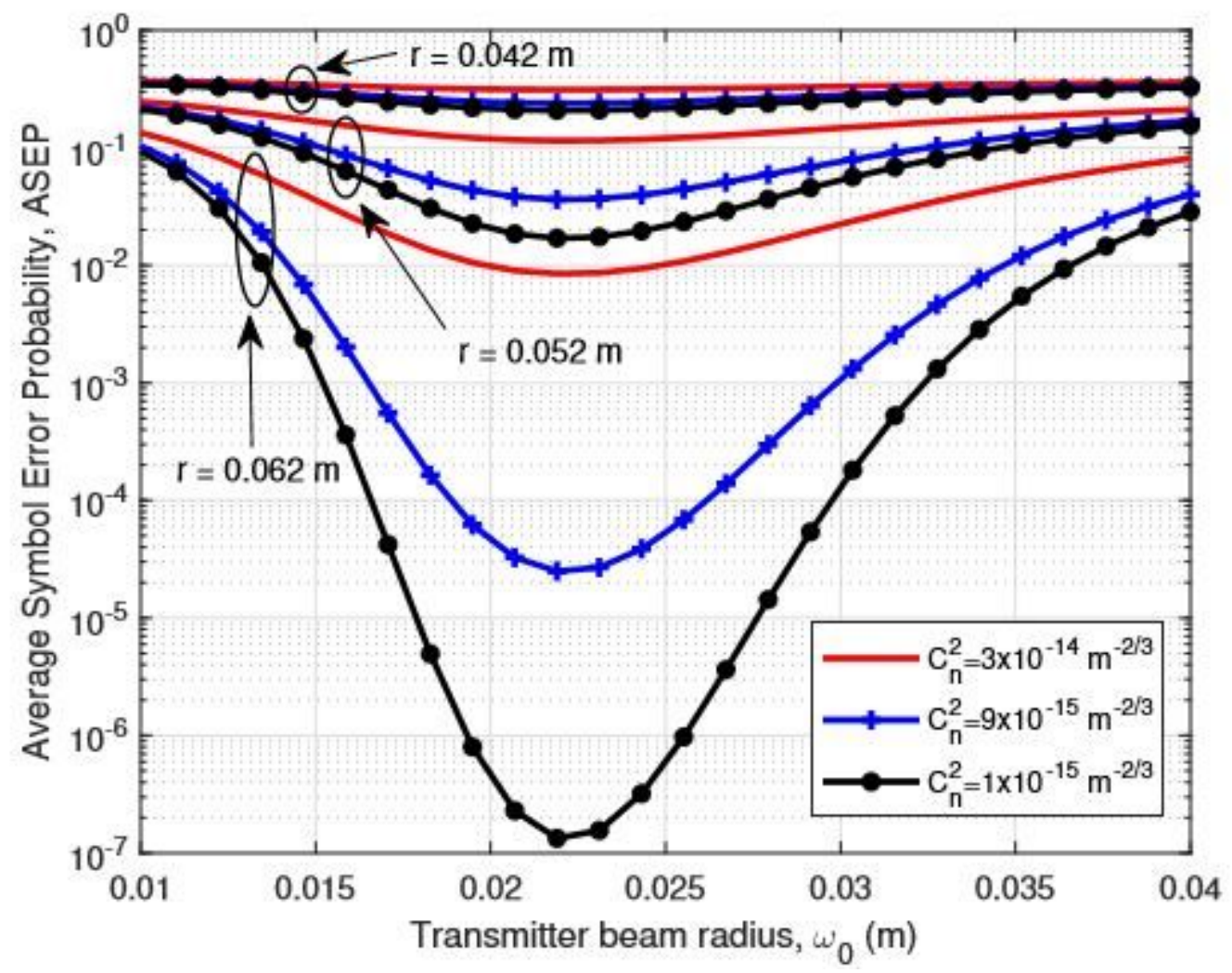




\section{Figure 9}

The ASEP performance against optical beamwidth $\mathrm{w}_{-} 0$ over various values of turbulent conditions $C_{-} n^{\wedge} 2$ and receiver aperture radius $r$ in Log-Normal with Rician P.E model employing P.E standard deviation $\sigma \_s$ $=0.07 \mathrm{~m}$, optical link displacement $\mathrm{z}=1000 \mathrm{~m}$ and average $\mathrm{SNR}=30 \mathrm{~dB}$.

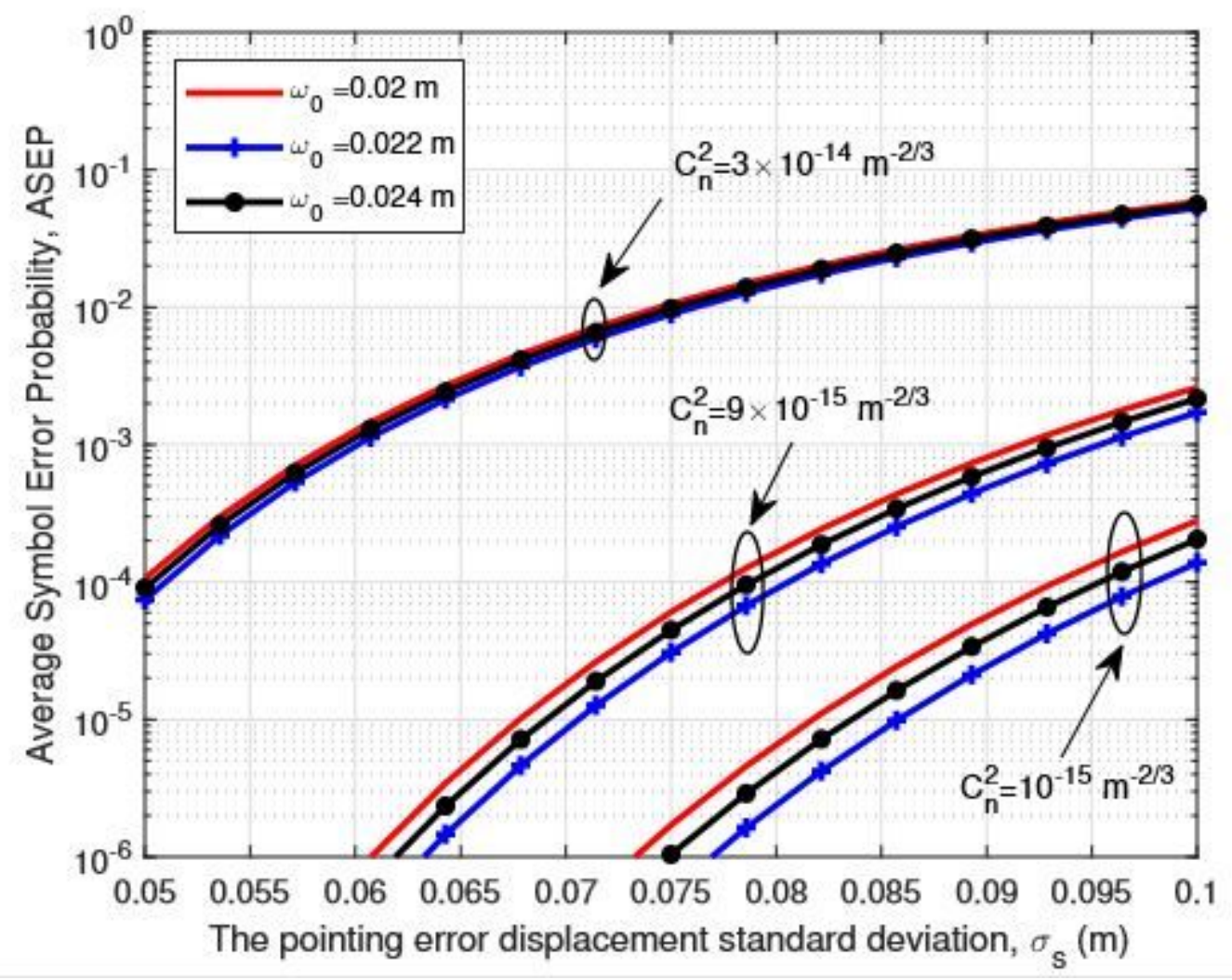

Figure 10

The ASEP performance against P.E displacement standard deviation $\sigma_{-} s$ over various values of turbulent conditions $C_{-} n^{\wedge} 2$ and optical beamwidth $w_{-} 0$ in Log-Normal with Rayleigh P.E model employing receiver aperture radius $r=0.052 \mathrm{~m}$, optical link displacement $\mathrm{z}=1000 \mathrm{~m}$ and average $\mathrm{SNR}=30 \mathrm{~dB}$. 


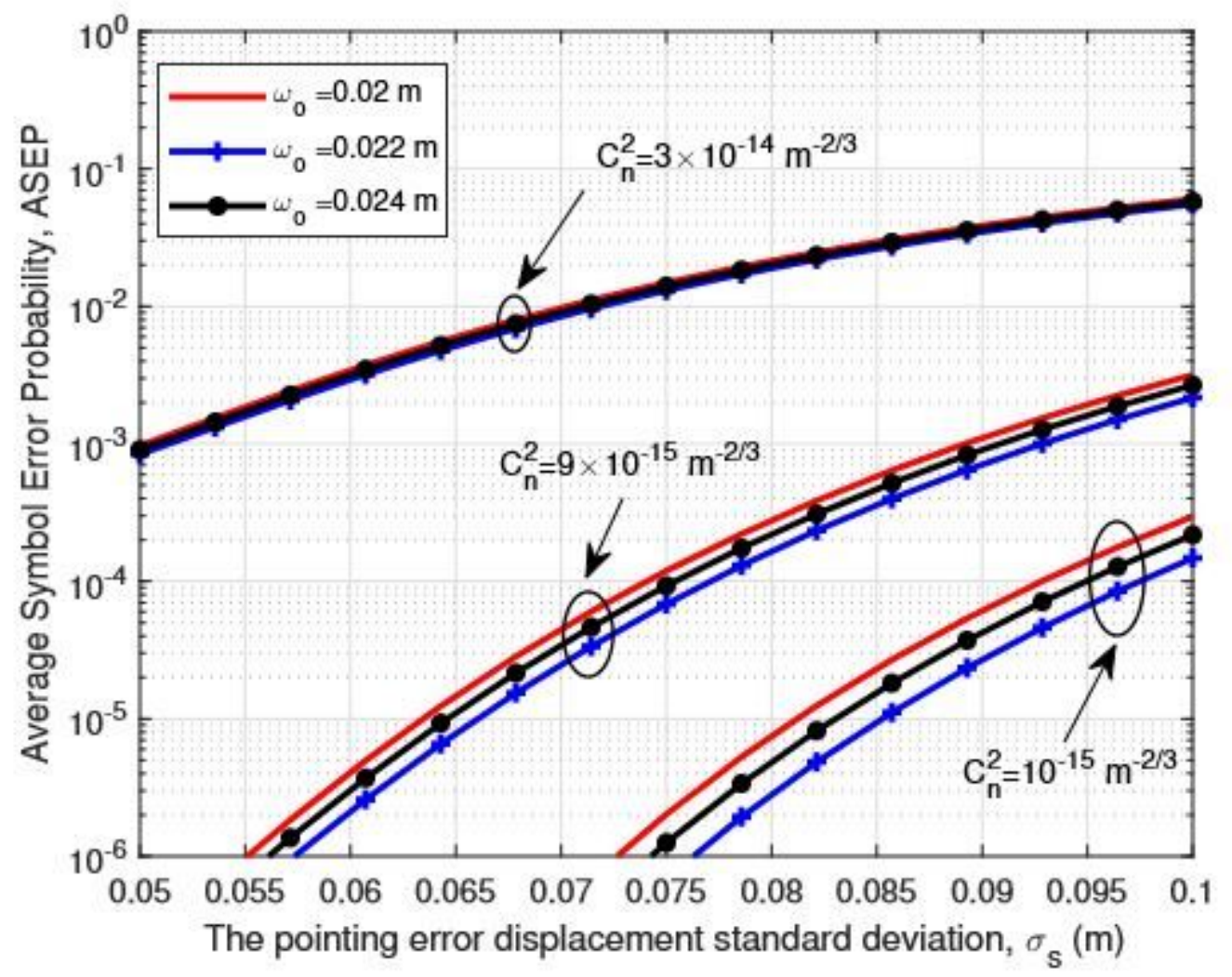

Figure 11

The ASEP performance against P.E displacement standard deviation $\sigma_{-}$s over various values of turbulent conditions $C_{-} n^{\wedge} 2$ and optical beamwidth $w_{-} 0$ in Log-Normal with Rician P.E model employing receiver aperture radius $r=0.052 \mathrm{~m}$, optical link displacement $\mathrm{z}=1000 \mathrm{~m}$ and average $\mathrm{SNR}=30 \mathrm{~dB}$.

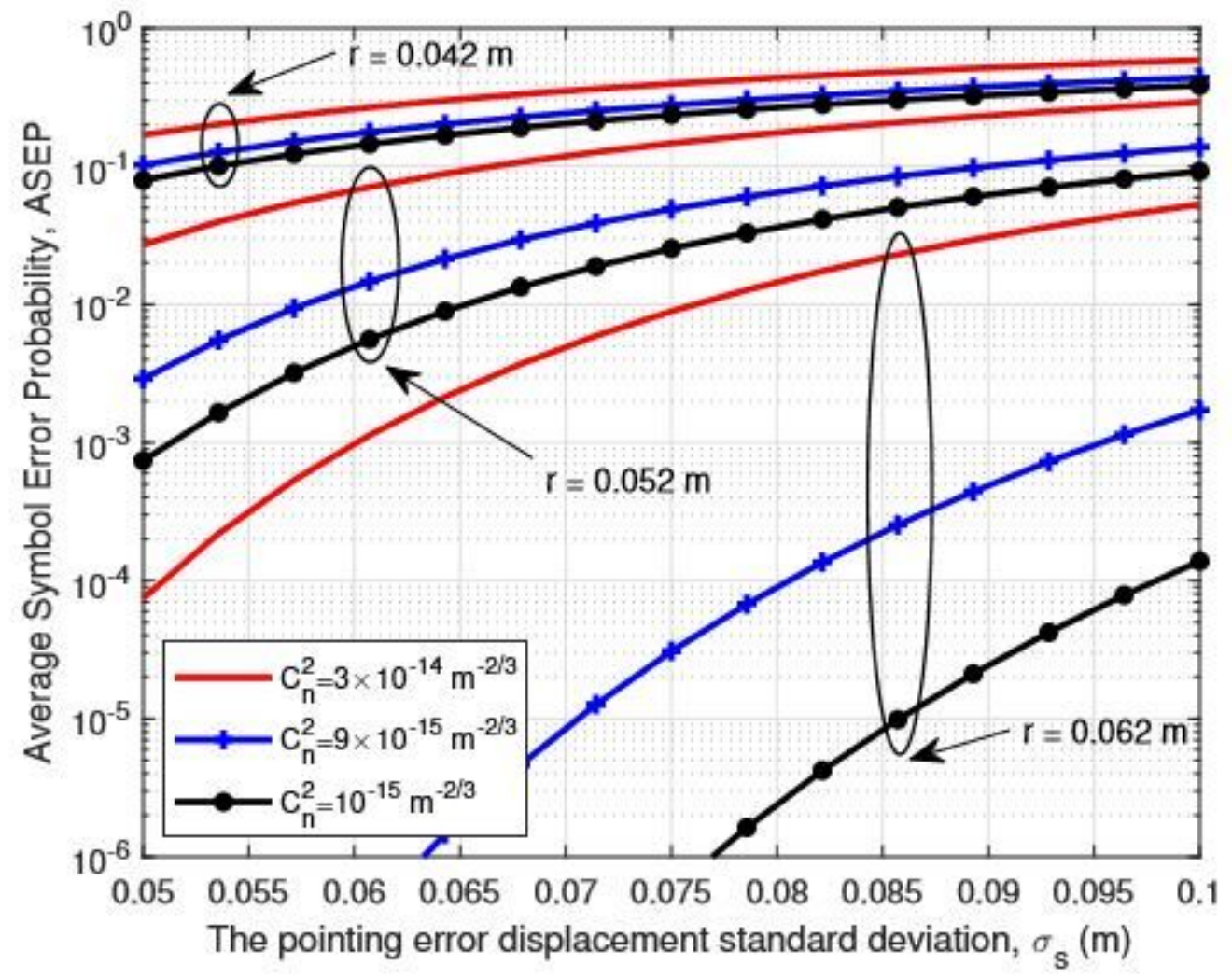


Figure 12

The ASEP performance against P.E displacement standard deviation $\sigma_{-}$s over various values of turbulent conditions $\mathrm{C} \_n^{\wedge} 2$ and receiver aperture radius $r$ in Log-Normal with Rayleigh P.E model employing optical beamwidth $w_{-} \_\approx 0.022 \mathrm{~m}$, optical link displacement $z=1000 \mathrm{~m}$ and average $S N R=30 \mathrm{~dB}$.

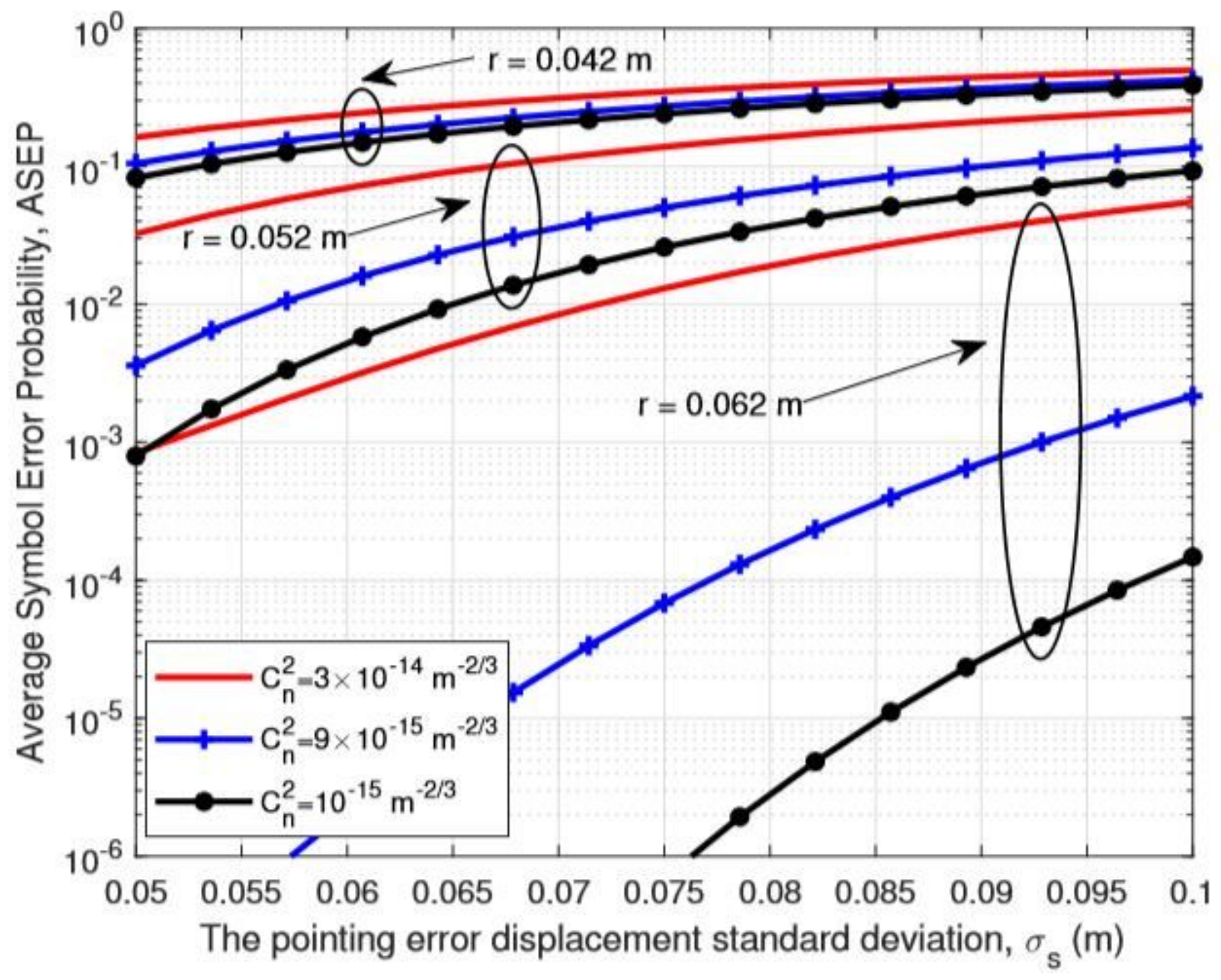

Figure 13

The ASEP performance against P.E displacement standard deviation $\sigma_{-}$s over various values of turbulent conditions $C_{-} n^{\wedge} 2$ and receiver aperture radius $r$ in Log-Normal with Rician P.E model employing optical beamwidth $w_{-} 0 \approx 0.022 \mathrm{~m}$, optical link displacement $\mathrm{z}=1000 \mathrm{~m}$ and average $S N R=30 \mathrm{~dB}$. 


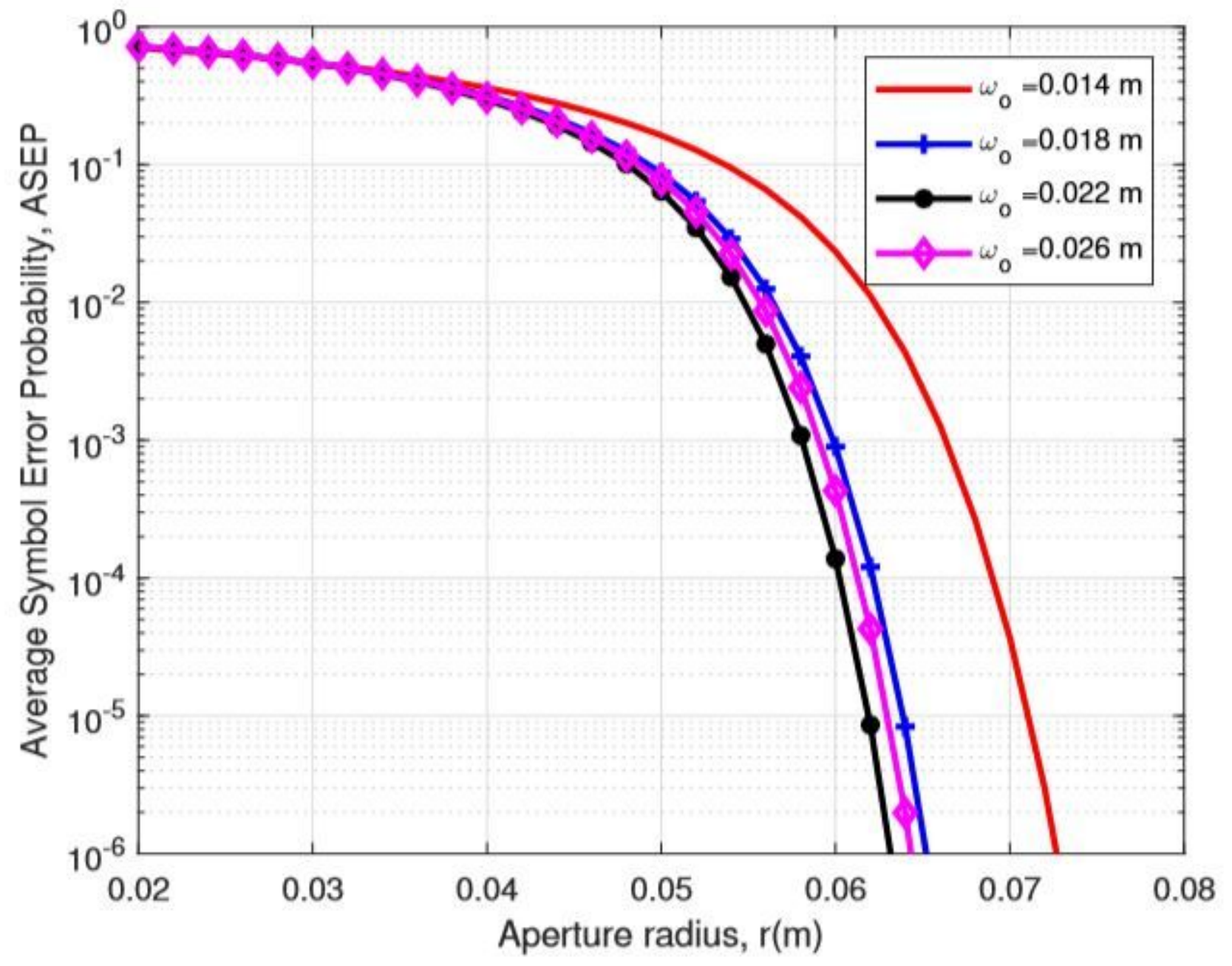

Figure 14

The ASEP performance against receiver aperture radius $r$ over various values of optical beamwidth $w \_0$ in Log-Normal with Rayleigh P.E model employing P.E displacement standard deviation $\sigma \_s=0.07 \mathrm{~m}$, optical link displacement $z=1000 \mathrm{~m}$ and average $S N R=30 \mathrm{~dB}$. 


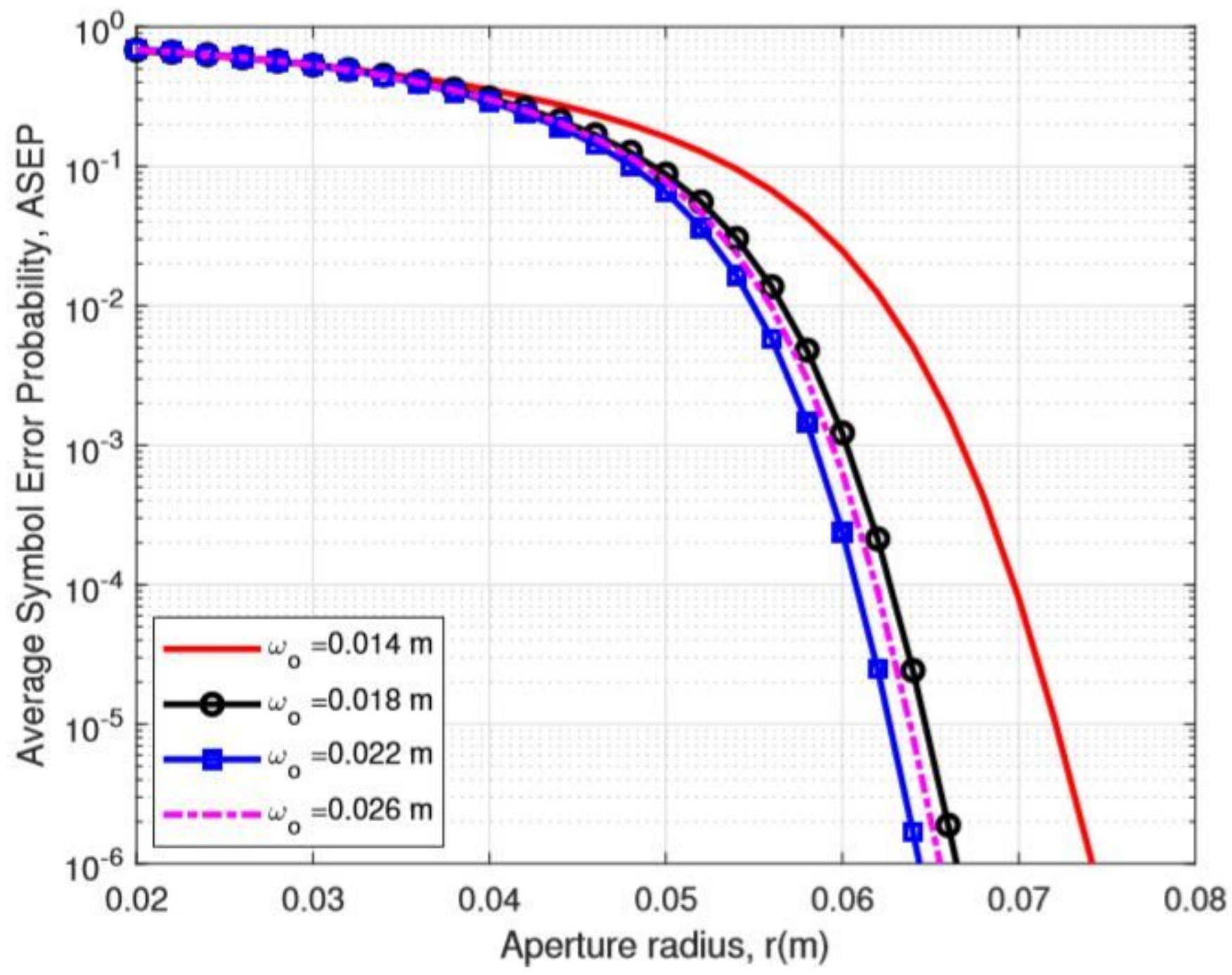

Figure 15

The ASEP performance against receiver aperture radius $r$ over various values of optical beamwidth $w_{-} 0$ in Log-Normal with Rician P.E model employing P.E displacement standard deviation $\sigma_{-} s=0.07 \mathrm{~m}$, optical link displacement $z=1000 \mathrm{~m}$ and average $S N R=30 \mathrm{~dB}$. 


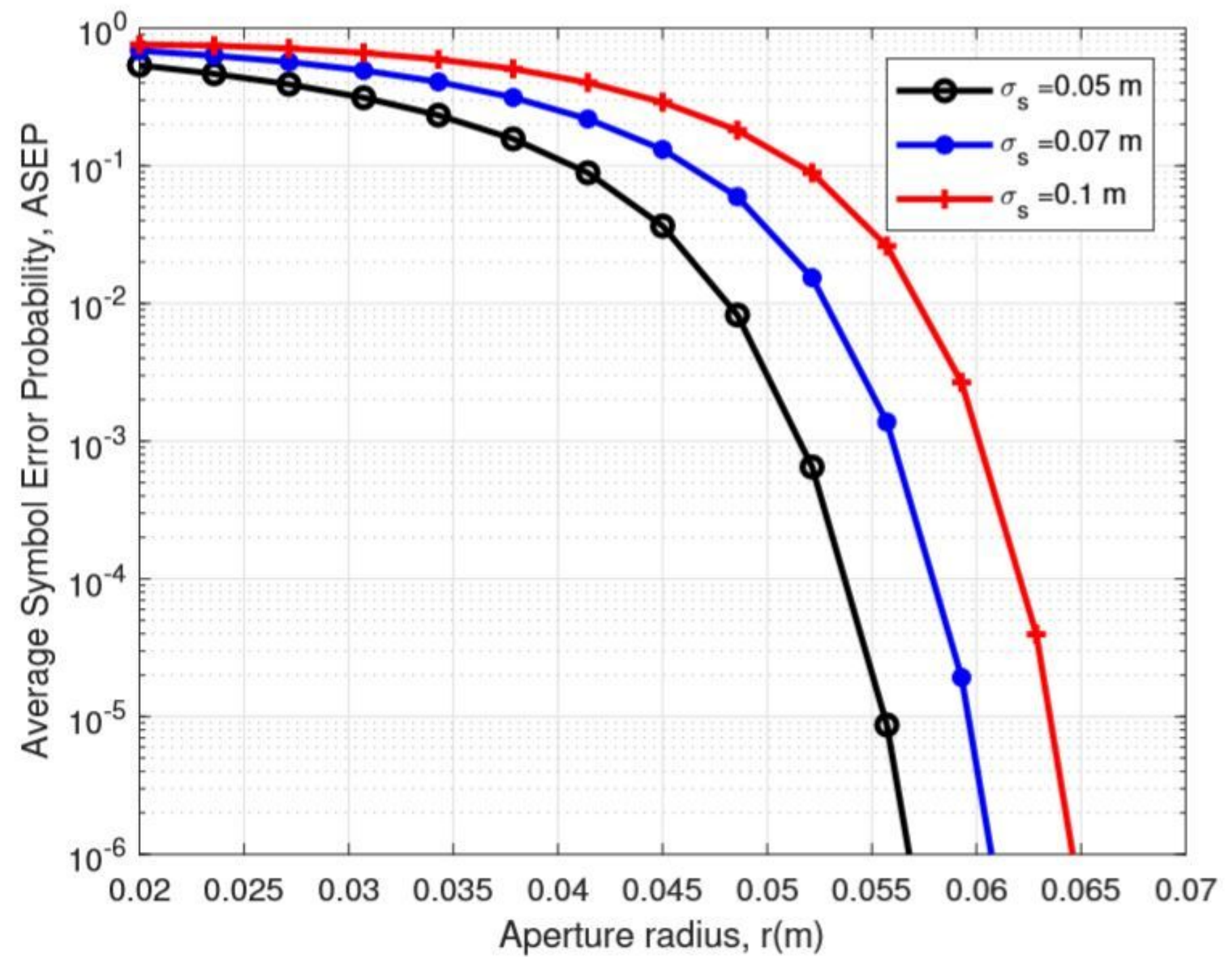

Figure 16

The ASEP performance against receiver aperture radius $r$ over various values of P.E displacement standard deviation $\sigma_{-} s$ in Log-Normal with Rayleigh P.E model employing optical beamwidth $\mathrm{w}_{-} 0=0.022 \mathrm{~m}$, optical link displacement $\mathrm{z}=1000 \mathrm{~m}$ and average $S N R=30 \mathrm{~dB}$. 


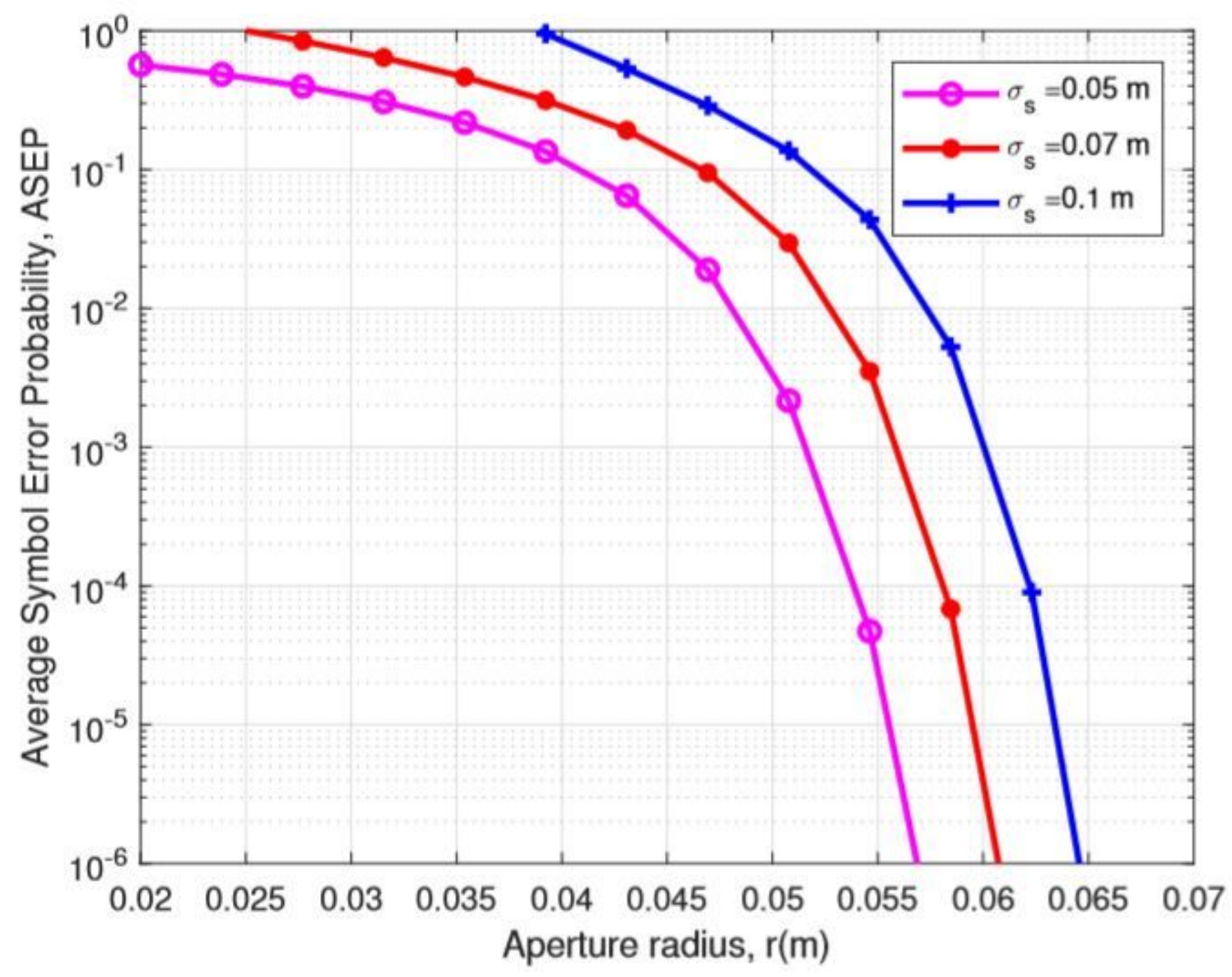

Figure 17

The ASEP performance against receiver aperture radius $r$ over various values of P.E displacement standard deviation $\sigma \_s$ in Log-Normal with Rician P.E model employing optical beamwidth $w_{-} 0=0.022 \mathrm{~m}$, optical link displacement $z=1000 \mathrm{~m}$ and average $S N R=30 \mathrm{~dB}$. 


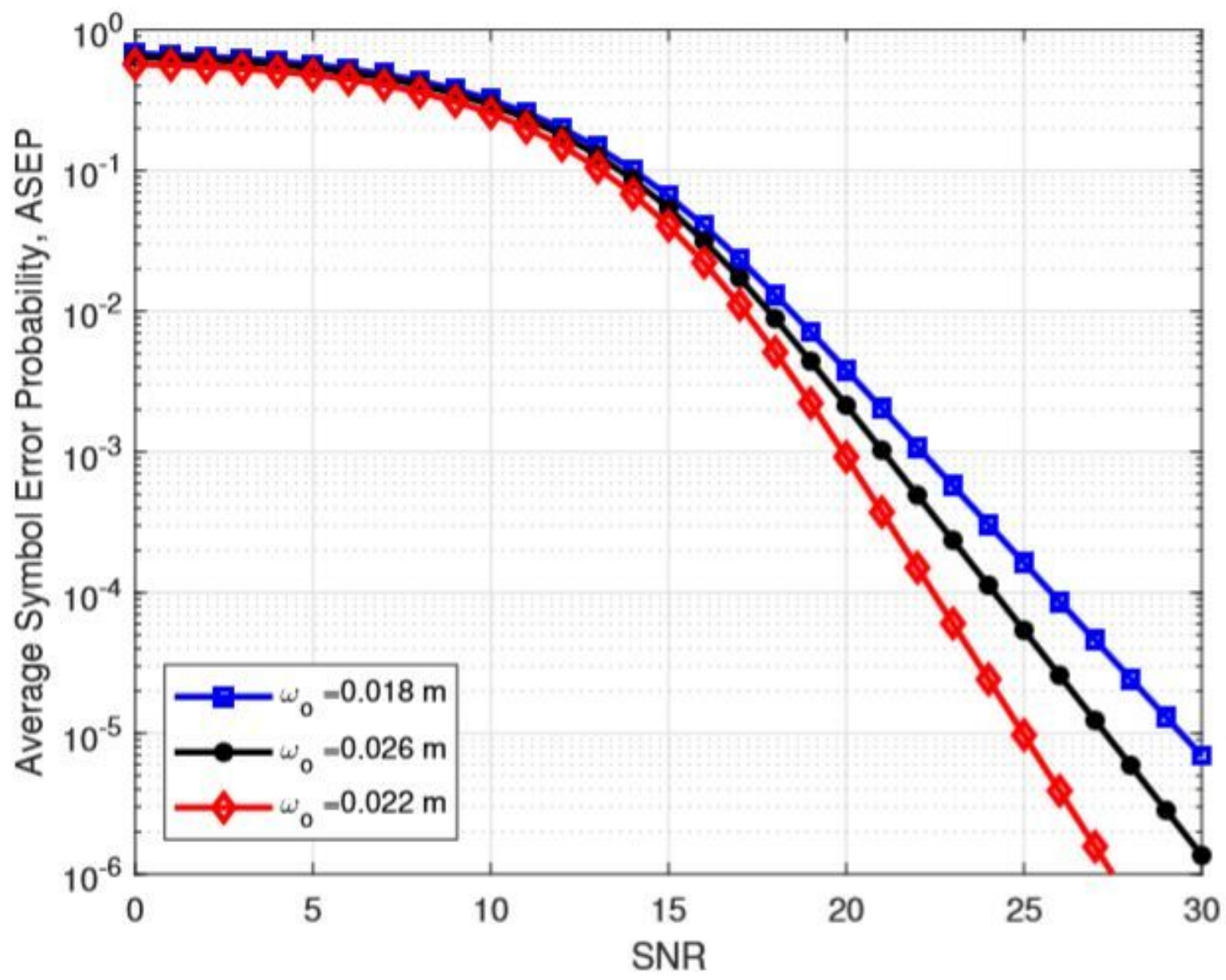

Figure 18

The ASEP performance against average SNR over various values of optical beamwidth $w_{-} 0$ in LogNormal with Rayleigh P.E model employing P.E displacement standard deviation $\sigma_{-} s=0.07 \mathrm{~m}$, optical link displacement $z=1000 \mathrm{~m}$ and receiver aperture radius $\mathrm{r}=0.052 \mathrm{~m}$. 


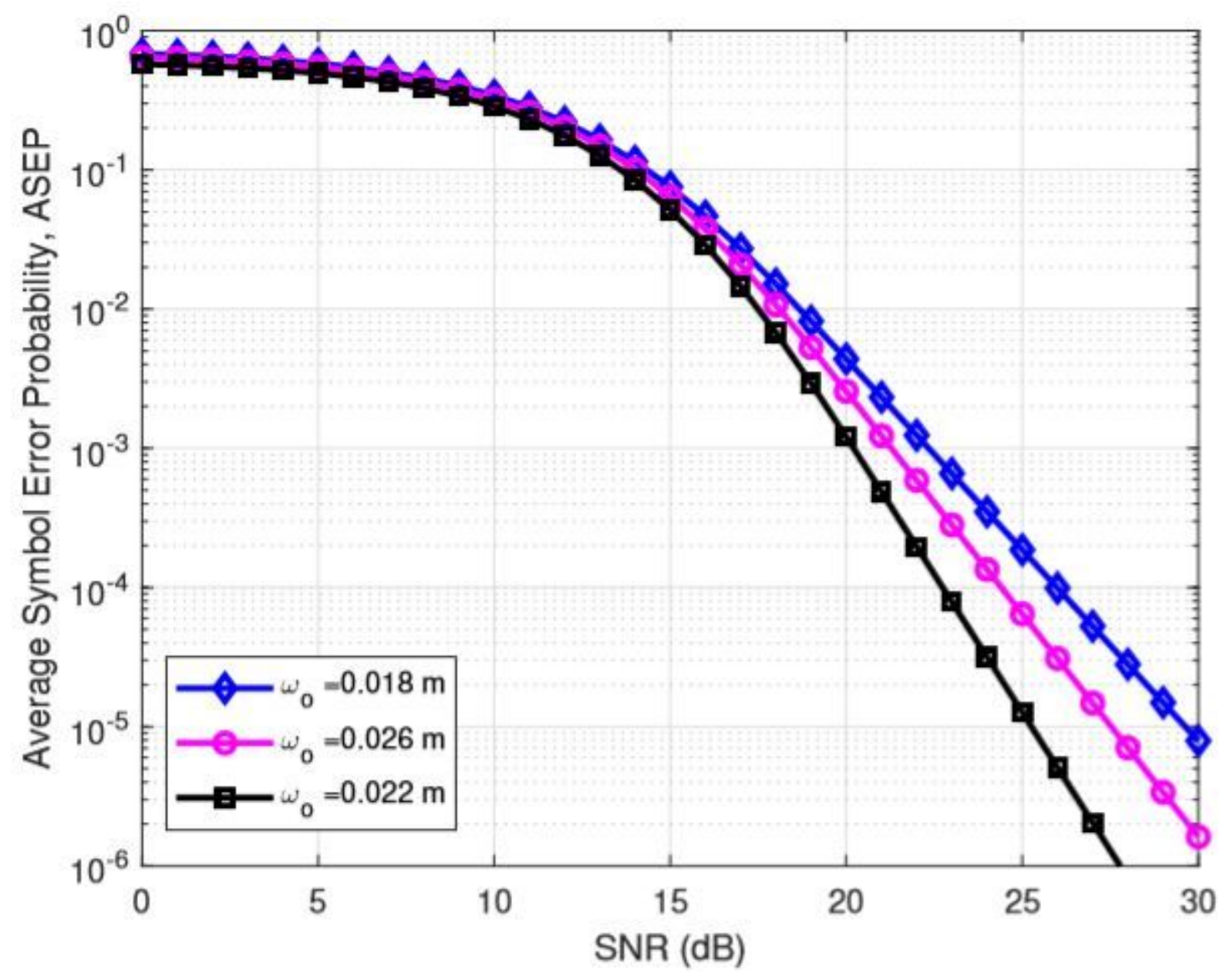

Figure 19

The ASEP performance against average SNR over various values of optical beamwidth $w_{-} 0$ in LogNormal with Rician P.E model employing P.E displacement standard deviation $\sigma_{-} \mathrm{s}=0.07 \mathrm{~m}$, optical link displacement $\mathrm{z}=1000 \mathrm{~m}$ and receiver aperture radius $\mathrm{r}=0.052 \mathrm{~m}$. 


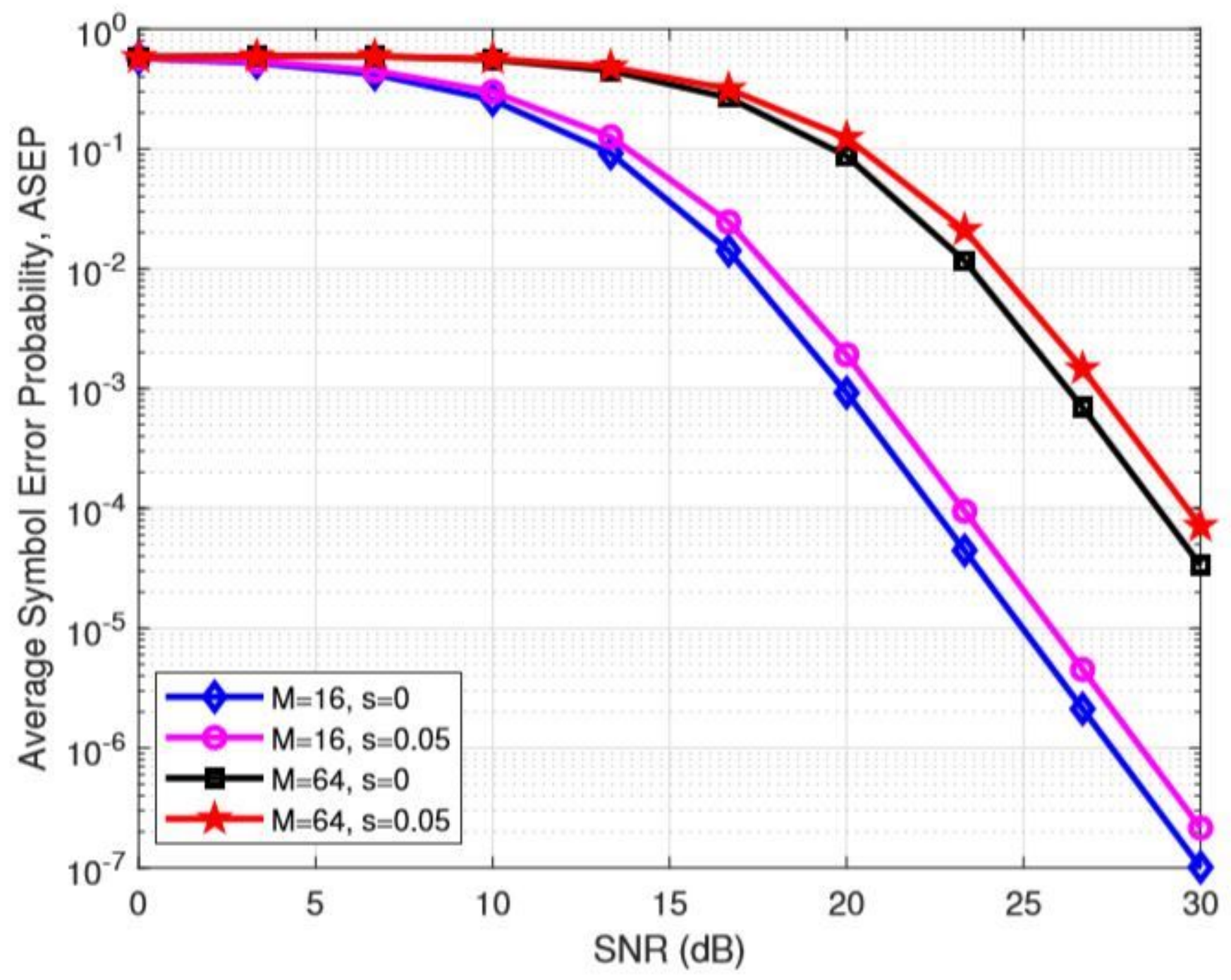

Figure 20

The M-TQAM $(M=16,64)$ ASEP performance against average SNR in Log-Normal with P.E model incorporating zero boresight $(\mathrm{s}=0)$ and nonzero boresight $(\mathrm{s}=0.05)$ employing P.E displacement standard deviation $\sigma \_s=0.07 \mathrm{~m}$, optical beamwidth $w_{-} 0 \approx 0.022 \mathrm{~m}$, turbulent condition $1 \times \otimes 10 \nabla^{\wedge}(-15) \mathrm{m}^{\wedge}(-2 / 3)$, optical link displacement $\mathrm{z}=1000 \mathrm{~m}$ and receiver aperture radius $\mathrm{r}=0.052 \mathrm{~m}$.

\section{Supplementary Files}

This is a list of supplementary files associated with this preprint. Click to download.

- Table1.PNG

- Table3.PNG

- Table2.PNG

- Table4.PNG 